\title{
MODELING OF SYNGAS REACTIONS AND HYDROGEN GENERATION OVER SULFIDES
}

Annual Technical Progress Report

September 18, 2002 - September 17, 2003

\author{
Kamil Klier - PI \\ Jeffery A. Spirko - Research Associate \\ Michael L. Neiman — Graduate Student \\ Issued March 2004 \\ U.S. Department of Energy \\ Research Project No. DE-FG26-01NT41276
Department of Chemistry
Lehigh University
6 E Packer Ave \\ Bethlehem, PA 18015-3173
}




\section{DISCLAIMER}

This report was prepared as an account of work sponsored by an agency of the United States Government. Neither the United States Government nor any agency thereof, nor any of their employees, makes any warranty, express or implied, or assumes any legal liability or responsibility for the accuracy, completeness, or usefulness of any information, apparatus, product, or process disclosed, or represents that its use would not infringe privately owned rights. Reference herein to any specific commercial product, process, or service by trade name, trademark, manufacturer, or otherwise does not necessarily constitute or imply its endorsement, recommendation, or favoring by the United States Government or any agency thereof. The views and opinions of authors expressed herein do not necessarily state or reflect those of the United States Government or any agency thereof. 


\section{ABSTRACT}

The objective of the research is to analyze pathways of reactions of hydrogen with oxides of carbon over sulfides, and to predict which characteristics of the sulfide catalyst (nature of metal, defect structure) give rise to the lowest barriers toward oxygenated hydrocarbon product. Reversal of these pathways entails the generation of hydrogen, which is also proposed for study.

In this second year of study, high-level density functional theory was used to model the edge defect of $\mathrm{MoS}_{2}$ both with and without $\mathrm{H}$ atoms and $\mathrm{H}_{2}$ molecules adsorbed on those edges. The structures and stabilities of the pure $\mathrm{MoS}_{2}$ edges were finalized and published [1]. Several $\mathrm{MoS}_{2}$ cluster models were examined to provide insight into the behavior of the more complex edges. With that knowledge, the adsorption locations, energies, and vibrational frequencies of $\mathrm{H}$ adsorption were determined. 


\section{Table of Contents}

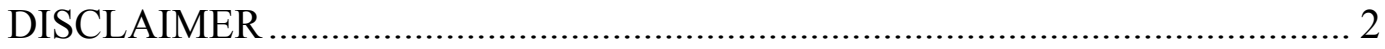

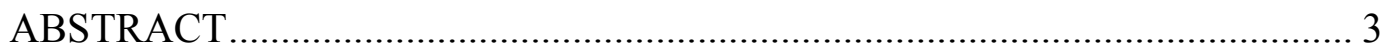

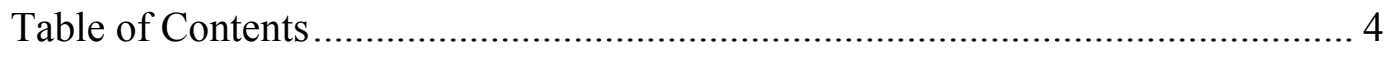

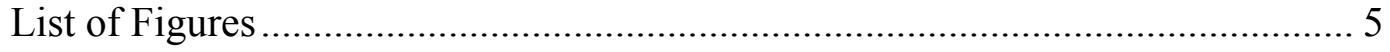

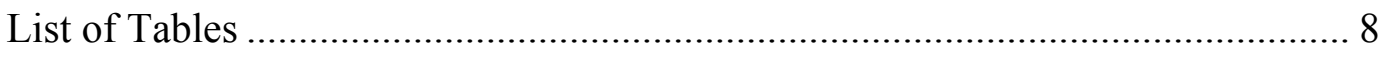

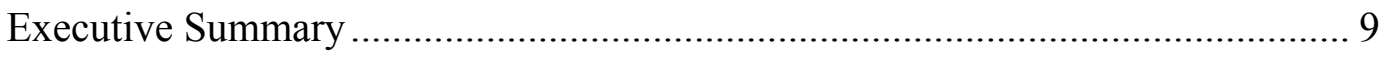

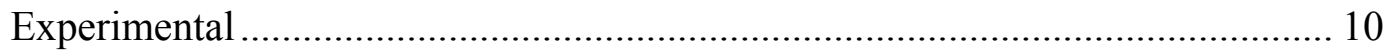

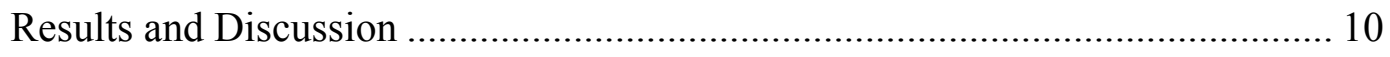

1. Objective and Background of the Research ................................................. 10

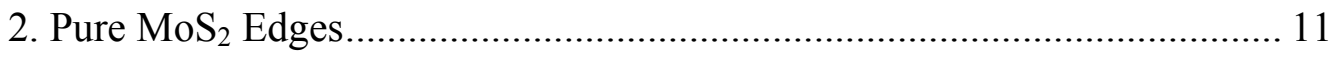

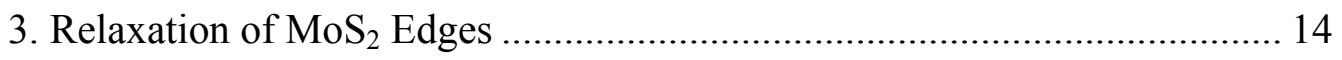

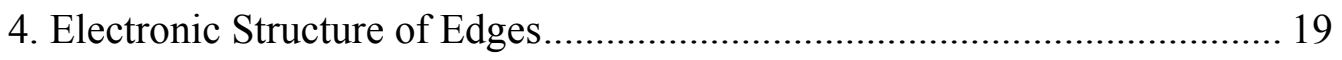

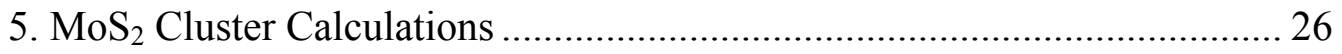

6. Cluster Calculations of $\mathrm{MoS}_{2}$ with Hydrogen................................................ 30

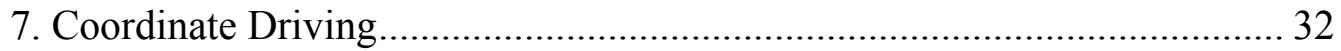

8. Orbital Analysis........................................................................................ 33

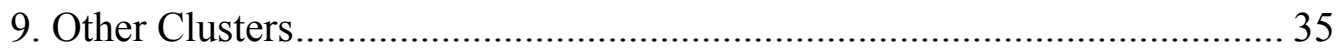

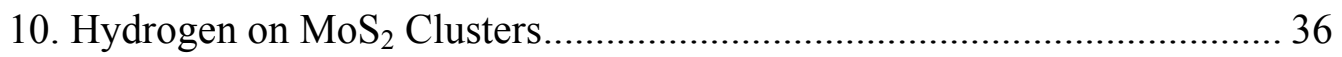

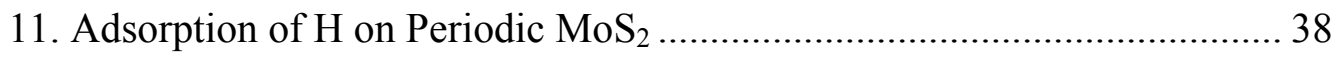

12. Thermodynamics of Hydrogen Adsorption................................................ 42

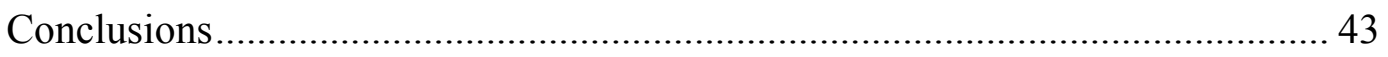

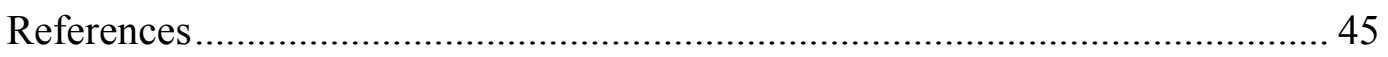




\section{List of Figures}

Figure 1: Picture of the $\mathrm{MoS}_{2}$ basal plane, as viewed from the top. Purple atoms are Mo, and yellow atoms are the $\mathrm{S}$ atoms which are above and below the Mo plane. One $(1 \overline{2} 10)$ cutting plane and the two possible $(10 \overline{1} 0)$ cutting planes are shown with black lines. The $(10 \overline{1} 0)$ a cut is more stable than the $(10 \overline{1} 0) b$ cut because it severs fewer bonds.

Figure 2: Side views along the unrelaxed $\mathrm{MoS}_{2}$ edges. Each figure shows one or more unit cells of a 4-MoS $\mathrm{M}_{2}$-unit wide ribbon which repeats into and out of the page, with the actual edges on the left and right sides.

Figure 3: Side views along the relaxed $\mathrm{MoS}_{2}$ edges. Each figure shows one or more unit cells of a 4-MoS $\mathrm{M}_{2}$-unit wide ribbon which repeats into and out of the page. The left and right sides of each view are the actual edges.

Figure 4: The two most stable relaxed edges. Here the coordination of the edge Mo and $\mathrm{S}$ atoms is easily seen. Some Mo-Mo distances and Mo-S bond lengths near the edges are shown. Those distances which are not marked were within $0.1 \AA$ of the lengths from the optimized 2-D sheet, $d(\mathrm{Mo}-\mathrm{Mo})=3.16 \AA$ and $d(\mathrm{Mo}-\mathrm{S})=2.42 \AA$. The two complementary sides of each edge are labeled.

Figure 5: A metastable geometry for the $(10 \overline{1} x)$ edge. The local minimum was obtained from a direct optimization of the unrelaxed geometry, while the true minimum in Figure 4 was derived from a slightly disordered unrelaxed geometry. When the symmetry was broken, the optimization procedure found the more stable configuration, leading to an energy $0.25 \mathrm{eV}$ per edge $\mathrm{MoS}_{2}$ unit lower than this symmetric configuration. Note that the coordination of the Mo atoms on the right is only 5 and the $\mathrm{S}$ atoms on the left have not assumed their up-down configuration seen in Figure 3.

Figure 6: Test of the convergence of $E_{\text {edge }}$ for two representative edges as the ribbon becomes wider. The $4 \times 2$ ribbon was sufficient according to this result. The energies are shown for the $(1 \overline{2} 10) \quad$ (solid), and $(1 \overline{2} 1 x) \quad$ (dashed) edges. .............. 18

Figure 7: Possible cleavage planes which would form "inclined edges" from a single $\mathrm{MoS}_{2}$ crystal.

Figure 8: Calculated valence band and conduction band DOS of the 2-D $\mathrm{MoS}_{2}$ sheet (black) and the most stable 1-D edges, $(1 \overline{2} 1 x)$ (blue) and (10 $\overline{1} x)$ (red). The DOS plots have been smoothed with a Gaussian having a FWHM of $0.5 \mathrm{eV}$. The HOMO energy in each case is marked with a vertical line.

Figure 9: Upper valence band structure of the 2-D $\mathrm{MoS}_{2}$ sheet. 21 
Figure 10: Valence band structure of the $(1 \overline{2} 1 x)$ and $(10 \overline{1} x)$ edge models of $\mathrm{MoS}_{2}$. The HOMO energies are marked with black lines. The HOMO of the $(10 \overline{1} x)$ edge was below the top of the valence band. Here $k_{y}$ is in units of $2 \pi / a$, where $a=6.32 \AA$ was the lattice constant for the $(10 \overline{1} x)$ edge and $a=5.473 \AA$ for the $(1 \overline{2} 1 x)$ edge.

Figure 11: Upper valence band structure of the $(1 \overline{2} 1 x)$ and $(10 \overline{1} x)$ edges of $\mathrm{MoS}_{2}$. The Fermi energies are marked with black lines which separate the HOMO from the LUMO. An electron in the HOMO of the $(10 \overline{1} x)$ edge was found to have an effective mass of $1.9 m_{e}$. Here $k_{y}$ is in units of $2 \pi / a$, where $a=6.32 \AA$ was the lattice constant for the $(10 \overline{1} x)$ edge and $a=5.473 \AA$ for the $(1 \overline{2} 1 x)$ edge............ 23

Figure 12: Experimentally derived band structure of $\mathrm{MoS}_{2}$ as determined by Böker [34] (circles) and the present calculation of the 2-D $\mathrm{MoS}_{2}$ sheet (black lines) and 3-D Bulk $\mathrm{MoS}_{2}$ (green lines).

Figure 13: Surface state of the Mo-exposed side of the $(10 \overline{1} x)$ edge. This orbital is the HOMO at the $\Gamma$-point. Clearly seen are the edge sulfur $p$ contributions, along with $\mathrm{d}$ orbitals of the edge Mo atoms aligned along the edge. The contributions of other atoms fall off as the distance from the edge increases. The orbital is cut off in the front and back by the walls of the repeating unit cell, leading to the white "hole" artifact seen in the ring of the Mo d orbital. 25

Figure 14: Initial (top) and relaxed (bottom) structures of $\left(\mathrm{MoS}_{2}\right)_{n}$ clusters, as viewed along the side resembling the $\mathrm{MoS}_{2}$ edges. The initial structures use the bond lengths and angles from the $\mathrm{MoS}_{2}$ crystal structure.

Figure 15: HOMO isosurfaces in singlet (top) and triplet (bottom) $\left(\mathrm{MoS}_{2}\right)_{n}$ clusters.... 27

Figure 16: Spin density isosurfaces in triplet $\left(\mathrm{MoS}_{2}\right)_{n}$ clusters for $n=1,3$, and 7 .

Figure 17: Stable and metastable geometries of $\mathrm{H}_{2}-\mathrm{MoS}_{2}$. Structure (a) is the ground state singlet dihydride, (b) is a metastable "tilted" configuration with an energy $0.3 \mathrm{eV}$ above the ground state, and (c) is the lowest-energy triplet $\eta^{2}$ hydrogen configuration, with an energy $0.6 \mathrm{eV}$ above the ground state. 31

Figure 18: Constrained optimization of the energy of the $\mathrm{H}_{2} \mathrm{MoS}_{2}$ molecule as a function of the $\mathrm{H}-\mathrm{Mo}-\mathrm{H}$ bond angle $\theta_{\mathrm{H}}$. The hydrogen molecule is attracted to the exposed Mo atom in the $\mathrm{MoS}_{2}$ monomer ( small $\theta_{\mathrm{H}}$ ) and could form a metastable $\eta^{2}-\mathrm{H}_{2}$ complex in a spin triplet. Overcoming a small activation energy leads to a stable configuration as a dihydride (large $\theta_{\mathrm{H}}$ ) in this coordinate-driven set of calculations. Easily seen are the metastable triplet state and a "tilted" singlet state, along with the ground singlet state. The upper singlet path maintains the $C_{2 v}$ symmetry, while the lower path breaks the symmetry to find the "tilted" configuration. 32 
Figure 19: Molecular orbitals of $\mathrm{H}_{2}-\mathrm{MoS}_{2}$ that have the highest $\mathrm{H}_{2} \sigma^{*}$ character for the optimized singlet (left) and triplet (right) configurations. Both orbitals show interaction between the $\sigma^{*}$ and the Mo dyz orbital, but in the triplet case, this MO is only singly occupied.

Figure 20: Energy levels of separated $\mathrm{H}_{2}$ and $\mathrm{MoS}_{2}$ monomer (left), the metastable triplet $\mathrm{H}_{2}-\mathrm{MoS}_{2}$ (center), and the ground state singlet $\mathrm{H}_{2}-\mathrm{MoS}_{2}$ (right). All geometries have the $C_{2 v}$ symmetry, and the orbitals are colored by their symmetries (black=A1; blue $=\mathrm{A} 2$; red $=\mathrm{B} 1$; green=B2). Between the triplet and singlet geometries, one A1 spin-up electron moves to the B1 spin-down orbital, a forbidden transition.

Figure 21: Geometries of the potassium-doped $\mathrm{MoS}_{2}$ and $\mathrm{NbS}_{2}$ monomers with $\mathrm{H}_{2}$ molecules attached to the transition metal. 35

Figure 22: The addition of potassium to the $\mathrm{NbS}_{2}$ molecule significantly decreases both the activation energy and the final adsorption energy of $\mathrm{H}_{2}$ on the $\mathrm{NbS}_{2}$ monomer. The pictured orbital is the majority-spin HOMO at the top of the barrier, which is a combination of the $\sigma^{*}$ orbital of $\mathrm{H}_{2}$ and the $\mathrm{d}_{x z}$ orbital of $\mathrm{Nb}$, with some contribution from the $\mathrm{S}$ atoms 36

Figure 23: Geometry of hydrogen adsorption on the $\mathrm{Mo}_{7} \mathrm{~S}_{14}$ cluster. The left side of the cluster resembles the $(10 \overline{1} x)$ edge of the $\mathrm{MoS}_{2}$ crystal and has monohydrides. The top and bottom resemble the $(10 \overline{1} 0)$ edge and show both $\eta^{2}-\mathrm{H}_{2}$ and dihydride

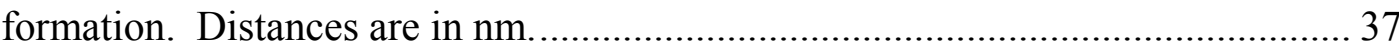

Figure 24: The most stable site for potassium adsorption on the $\mathrm{Mo}_{7} \mathrm{~S}_{14}$ cluster. The dihydrides on the Mo atoms nearest the $\mathrm{K}$ are stabilized by the addition of $\mathrm{K}$....... 38

Figure 25: Geometries of adsorption locations for hydrogen atoms on the $(10 \overline{1} x)$ and $(1 \overline{2} 1 x)$ edges of $\mathrm{MoS}_{2}$. When two $\mathrm{H}$ atoms are seen, it is because of the repeating unit cell. 40

Figure 26: Geometries tested with two $\mathrm{H}$ atoms adsorbed on the $\mathrm{MoS}_{2}$ edge 42 


\section{List of Tables}

Table 1: Unrelaxed energies of the various $\mathrm{MoS}_{2}$ edges. All calculations were done with $4 \times 2$ unit cells, and the energy per unit cell of the 2-D $\mathrm{MoS}_{2}$ sheet was $\mu=$ $-23538.08 \mathrm{eV}$. The edges are listed in the same order as in Table 2.

Table 2: Relaxed energies of the various $\mathrm{MoS}_{2}$ edges, measured per unit cell of the ribbon. All calculations were done with $4 \times 2$ unit cells. The large relaxation energy of the $(10 \overline{1} x)$ edge is due to reconstruction of the $\mathrm{S}$ atoms along that edge.

Table 3: Valence-band peak locations (eV) from experiment [31] and the present calculations. The peaks are labeled by binding energy from the least to the most bound. The energies of the present calculation have been shifted so that the energy of peak A matches.

Table 4: Energies of formation $\Delta E_{\mathrm{f}}$, relaxation $\Delta E_{\mathrm{r}}$, and of the triplet and singlet states in the $\left(\mathrm{MoS}_{2}\right)_{n}$ clusters. All energies are expressed in $\mathrm{eV} / \mathrm{MoS}_{2}$ unit

Table 5: Various properties for the most stable singlet and triplet geometries of the $\mathrm{H}_{2}-$ $\mathrm{MoS}_{2}$ complex.

Table 6: Adsorption energies of hydrogen atoms on $\mathrm{MoS}_{2}(10 \overline{1} x)$ and $(1 \overline{2} 1 x)$ edges. The binding energy of the $\mathrm{H}_{2}$ molecule is $-2.28 \mathrm{eV} /$ atom. The adsorption of hydrogen on the $\mathrm{MoS}_{2}$ basal plane is shown at the bottom. The labels of the sides are defined in Figure 4.

Table 7: Adsorption energies and vibrational frequencies for some $2 \mathrm{H}-\left(\mathrm{MoS}_{2}\right)_{n}$ configurations. $\Delta E=\mathrm{E}\left(2 \mathrm{H}-\left(\mathrm{MoS}_{2}\right)_{n}\right)-\left[\mathrm{E}\left(\left(\mathrm{MoS}_{2}\right)_{n}\right)+\mathrm{E}\left(\mathrm{H}_{2}\right)\right]$ 


\section{Executive Summary}

Stability of the $\mathrm{MoS}_{2}$ edges was found to be in the order $(10 \overline{1} x)($ most stable $)>(1 \overline{2} 1 x)>(1 \overline{2} 10)>(10 \overline{1} 0)$, where the inclination index $x=3$ or 4 . A large relaxation energy was associated with the reconstruction of the edge from ideal geometry of a cut through the $2 \mathrm{H}-\mathrm{MoS}_{2}$ crystal: for the $(10 \overline{1} x)$ edge, energy of $0.63 \mathrm{eV}$ per $\mathrm{MoS}_{2}$ molecular formula was released upon a concerted movement of exposed $\mathrm{S}$ atoms that increased the coordination of the edge Mo atoms from 4 to 5. The electronic band structure calculation identified surface states, which had a metallic character for the $(10 \overline{1} x)$ edge and a narrow gap semiconducting character for the $(1 \overline{2} 1 x)$ edge. For reference purposes, periodic band structure calculation of a single 2-D sheet of $\mathrm{MoS}_{2}$ by the LCAO DFT method used in this work yielded results practically identical to earlier LAPW DFT calculations of Park et al. [2]. The HOMO of the $(10 \overline{1} x)$ edge was found to be a surface state that penetrates beyond the first layer of edge Mo atoms. The effective mass of an electron in this state was calculated to be $1.9 m_{e}$, compared to $4.1 m_{e}$ for a hole in the HOMO of the 2-D sheet. Clusters of $\left(\mathrm{MoS}_{2}\right)_{n}(n=7,3,2,1)$ also showed large relaxation energies from the $2 \mathrm{H}-\mathrm{MoS}_{2}$ geometry, with the main tendency to increase the coordination of the exposed Mo atoms by inward movement. The difference in energies of the spin singlet and triplet states increased from near zero for $\left(\mathrm{MoS}_{2}\right)_{7}$ to $0.53 \mathrm{eV}$ for the single $\mathrm{MoS}_{2}$ molecule, in excellent agreement with the data for $\mathrm{MoS}_{2}$ in frozen argon matrix reported by Liang and Andrews [3].

The edges of $\mathrm{MoS}_{2}$ were tested for activation of hydrogen using density functional theory by modeling the molybdenum disulfide monomer, small clusters, and periodic edge models. Attachment of $\mathrm{H}_{2}$ to the $\mathrm{MoS}_{2}$ molecule was studied in detail, and several different structures were found. A dihydride with the hydrogen atoms far apart was most stable. In this configuration, the $\sigma^{*}$ orbital of the $\mathrm{H}_{2}$ mixed with the d orbitals of Mo to achieve the hydrogen activation. The less stable $\eta^{2}$ hydrogen configuration had the $\mathrm{H}_{2}$ bond slightly stretched with the $\mathrm{H}$ atoms equidistant from the Mo center. Addition of a potassium dopant further stabilized the attachment of the $\mathrm{H}_{2}$. A triangular $\mathrm{Mo}_{7} \mathrm{~S}_{14}$ cluster provided a small model with edges resembling those of the cut $\mathrm{MoS}_{2}$ sheets. On this cluster, both the $\eta^{2}$ hydrogen and the dihydride were seen. Various positions for atomic hydrogen adsorption on the most stable periodic $\mathrm{MoS}_{2}$ edges were tested and the vibrational frequencies were calculated. In addition, some configurations with $\mathrm{H}_{2}$ adsorption on the edges were modeled. 


\section{Experimental}

In this theoretical modeling effort, the results were obtained using the following DFT $[4,5]$ methods:

A. Periodic generalized gradient approximation (GGA) LCAO method with the DNP basis set and the Perdew-Wang '91 (PW) functional [6] as embedded in the $\mathrm{DMol}^{3}$ package [7];

B. Periodic generalized gradient approximation (GGA) FP-LAPW method with a dual planewave/spherical harmonics basis set and the Perdew-Burke-Ernzerhof '96 (PBE) functional [8] as embedded in the Wien2k code [9];

C. Generalized gradient approximation (GGA) LCAO method with the $\mathrm{DN}^{* *}$ basis set and the Becke-Perdew functional $[10,11]$ as embedded in the Spartan package [12];

D. Semiempirical methods for preoptimization of geometry.

Geometry optimizations used the Broyden-Fletcher-Goldfarb-Shanno (BFGS) [13] Hessian updating scheme, starting with the ideal bulk-derived $\mathrm{MoS}_{2}$ coordinates. At times, the initial positions of some atoms were shifted slightly to break symmetry and allow the structure to find its natural minimum.

Benchmarking and comparison of the various methods were also performed. We find the semiempirical pm3(tm) code [14] particularly deficient by arriving at totally wrong geometries, presumably due to a bad parametrization for molybdenum, a key element in our systems. All other properties obtained with this method are of course also in doubt. In contrast, our experience with methods A-C above $[2,15,16,17,18,19]$ permits the conclusion that results based on these methodologies are valid and reliable, albeit always amenable to refinement.

One particular challenge is to account for weak interactions such as those between adjacent $\mathrm{MoS}_{2}$ sheets across the Van der Waals gap. A successful calculation by the periodic full-potential linearized augmented plane wave (FP-LAPW) DFT method was reported last year to yield the weak attraction $(0.028 \mathrm{eV} / \mathrm{MoS} 2 \mathrm{unit})$, albeit the calculated attractive energy still differs from the experimental value of $0.013 \mathrm{eV} / \mathrm{MoS} 2$ unit and the calculated size of the Van der Waals gap was larger than the experimental value.

\section{Results and Discussion}

\section{Objective and Background of the Research}

Overall objective. The objective of this research is to analyze pathways of reactions of hydrogen with oxides of carbon over sulfides, and predict which characteristics of the sulfide catalyst give rise to the lowest barriers toward oxygenated 
hydrocarbon product. The present research aims at providing complementary and predictive theoretical background for an economical optimization of these processes.

Methodology. Molecular conversions at interfaces occur via a flow of electrons during movement of atomic nuclei from reactant to product configurations over energy barriers the magnitude of which determine the reaction rate. Reactants $(\mathrm{R})$ and products (P) are at local minima of a multidimensional energy-coordinate space, and the barrier is at a saddle point of that space called the Transition State (TS). The points R, TS and P are the stationary points of the reaction pathway, and search for these points is carried out by optimization methods of quantum mechanics. Specific methods used in this work are listed as A-D in the Experimental section of this report. Development of codes for TS searches in periodic systems is also planned. An important aspect of the modeling is evaluation of reliability and accuracy of the methods used.

\section{Pure $\mathrm{MoS}_{2}$ Edges}

To date, published DFT calculations of $\mathrm{MoS}_{2}$ edges have focused on the (10 $\left.\overline{1} 0\right)$ edges $[20,21,22,23,24,25,26,27,28,29,30]$. These are not, however, the only stable edge planes. Figure 1 shows the $\mathrm{MoS}_{2}$ basal plane, with three possible cutting planes marked. There are two $(10 \overline{1} 0)$ planes, and one $(1 \overline{2} 10)$ plane. In addition, the sheet can be cut at an angle, leading to stoichiometric unit cells with partial sulfur coverage on both sides. We refer to these edges as $(10 \overline{1} x)$ and $(1 \overline{2} 1 x)$. There is only one $(10 \overline{1} x)$ cut. The unrelaxed edges are shown in Figure 2.

All edge calculations reported herein used 1-D ribbon models with $\omega \times \ell$ rectangular supercells cut from 2-D $\mathrm{MoS}_{2}$ sheets having a rhombohedral unit cell with the experimental lattice dimensions $a=b=3.16 \AA$. Sheets were separated by $40 \AA$ in the 2$\mathrm{D}$ model, and the distance from the Mo-plane to the S-plane was $1.587 \AA$. The energy of the sheet was $\mu=-23538.08 \mathrm{eV}$ per $\mathrm{MoS}_{2}$ unit. The edge supercells were $\ell=2 \mathrm{MoS}_{2}$ units long along the edge, while the width $\omega$ of the ribbons from edge to counter-edge, was varied from $\omega=2$ to $5 \mathrm{MoS}_{2}$ units. Tests for convergence showed that $\omega=4$ was sufficient to ensure that interactions between the edges of the same ribbon are minimized, so $4 \times 2$ unit cells were used in most calculations. The ribbons were spaced $40 \AA$ apart in the non-repeating directions to eliminate interactions between neighboring cells. For our $\omega \times 2$ cells, the cell length was $6.32 \AA$ for the $(10 \overline{1}$.) edges and $5.473 \AA$ for the $(1 \overline{2} 1$.) edges.

The energy per $\mathrm{MoS}_{2}$ unit of an edge in the atomic geometry $\mathbf{R}$ was calculated directly from the total energy $E_{\text {tot }}(\mathbf{R})$ of the 1-D ribbon unit cell:

$$
E_{\text {edge }}(\mathbf{R})=\frac{1}{2 \ell}\left(E_{\text {tot }}(\mathbf{R})-N \mu\right)
$$

where $\mu$ is the energy per molecular unit of the 2-D $\mathrm{MoS}_{2}$ sheet and $N=\omega \ell$ is the number of $\mathrm{MoS}_{2}$ units in the ribbon unit cell. The factor of $\frac{1}{2}$ entered because both the edge and 
counter-edge are involved. As defined, $E_{\text {edge }}(\mathbf{R})$ is the average energy of the two edges. In the $(1 \overline{2} 10)$ case where the edge and counter-edge are identical, $E_{\text {edge }}(\mathbf{R})$ is the absolute edge energy per $\mathrm{MoS}_{2}$ unit.

Energies for both the unrelaxed and relaxed configurations were calculated, and the relaxation energy was defined as the change in edge energy from the initial to the relaxed configuration:

$$
\Delta E_{\mathrm{r}}=E_{\text {edge }}(\text { relaxed })-E_{\text {edge }}(\text { unrelaxed })
$$

The relaxation energy $\Delta E_{\mathrm{r}}$ is always negative.

The unrelaxed energies derived from the bulk atomic positions are listed in Table 1. The relaxation energy playd a significant role in determining the energy of the edge, so the unrelaxed energies do not determine the overall stability.

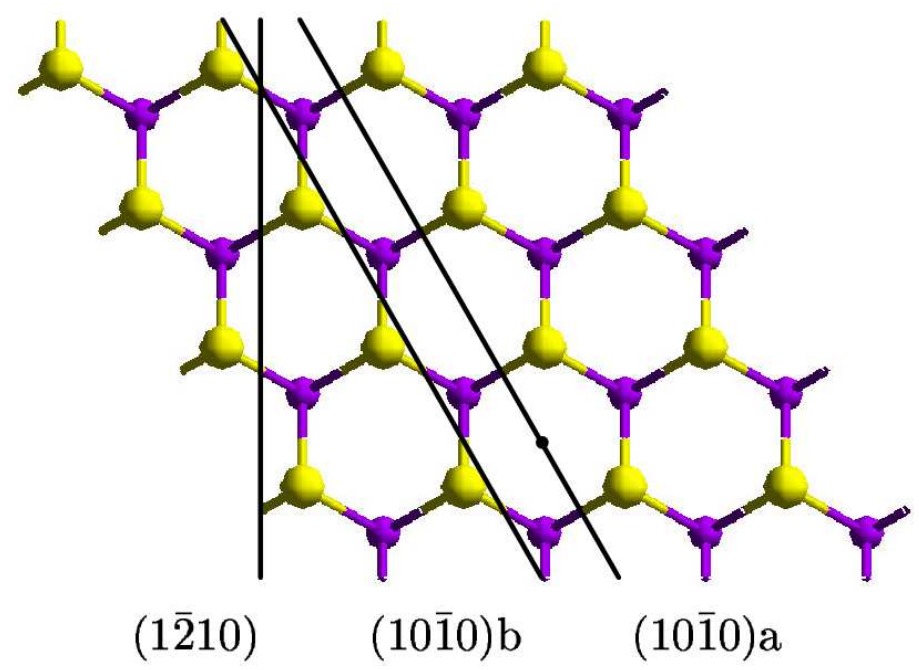

Figure 1: Picture of the $\mathrm{MoS}_{2}$ basal plane, as viewed from the top. Purple atoms are $\mathrm{Mo}$, and yellow atoms are the $\mathrm{S}$ atoms which are above and below the Mo plane. One $(1 \overline{2} 10)$ cutting plane and the two possible $(10 \overline{1} 0)$ cutting planes are shown with black lines. The $(10 \overline{1} 0) a$ cut is more stable than the $(10 \overline{1} 0) b$ cut because it severs fewer bonds. 
Table 1: Unrelaxed energies of the various $\mathrm{MoS}_{2}$ edges. All calculations were done with $4 \times 2$ unit cells, and the energy per unit cell of the 2-D $\mathrm{MoS}_{2}$ sheet was $\mu=-23538.08 \mathrm{eV}$. The edges are listed in the same order as in Table 2.

\begin{tabular}{ccc}
\hline Edge & $\begin{array}{c}E_{\text {tot }}(\text { unrelaxed }) \\
(\mathrm{eV})\end{array}$ & $\begin{array}{c}E_{\text {edge }}(\text { unrelaxed }) \\
\left(\mathrm{eV} / \mathrm{MoS}_{2} \text { unit }\right)\end{array}$ \\
\hline$(10 \overline{1} 0) \mathrm{b}$ & -188287.45 & 4.30 \\
$(10 \overline{1} 0) \mathrm{a}$ & -188295.65 & 2.25 \\
$(1 \overline{2} 10)$ & -188296.13 & 2.13 \\
$(1 \overline{2} 1 x)$ & -188294.92 & 2.43 \\
$(10 \overline{1} x)$ & -188293.71 & 2.73 \\
\hline
\end{tabular}

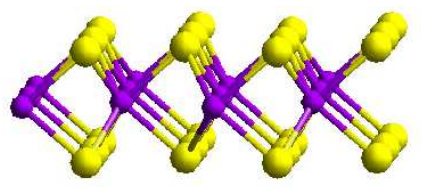

$(10 \overline{1} 0) \mathrm{b}$

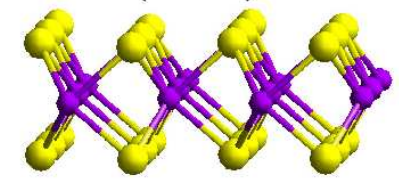

$(10 \overline{1} 0) \mathrm{a}$

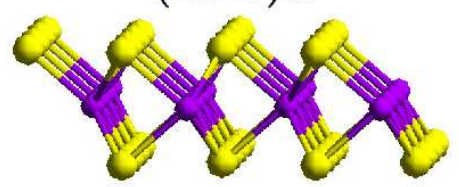

$(10 \overline{1} x)$

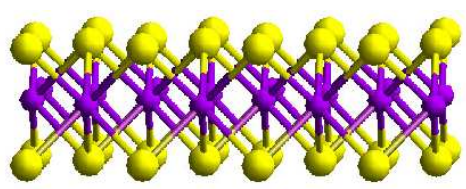

$(1 \overline{2} 10)$

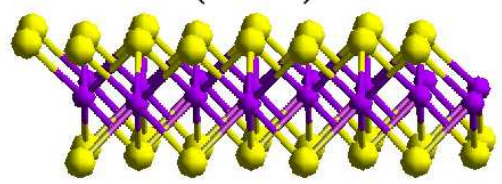

$(1 \overline{2} 1 x)$

Figure 2: Side views along the unrelaxed $\mathrm{MoS}_{2}$ edges. Each figure shows one or more unit cells of a 4-MoS 2 -unit wide ribbon which repeats into and out of the page, with the actual edges on the left and right sides. 


\section{Relaxation of $\mathrm{MoS}_{2}$ Edges}

The relaxation of the $\mathrm{MoS}_{2}$ edges produced the geometries shown in Figures 3 and 4. The energies $E_{\text {edge }}$ (relaxed) are given in Table 2. The (101 $\left.x\right)$ edge was found to be the most stable, with the $(1 \overline{2} 1 x)$ edge being only $0.09 \mathrm{eV} / \mathrm{MoS}_{2}$ unit higher in energy.

Upon relaxation, the edges exhibited various behaviors. In the three "verticallycut" edges, the exposed Mo atoms relaxed inward, while the $\mathrm{S}$ atoms moved out from the edge and in toward the Mo plane. The S-Mo-S bond angle was $82.05^{\circ}$ in the optimized 2-D sheet. On the Mo-exposed side of the (10 $\overline{1} 0)$ a edge, this angle increased to $83.7^{\circ}$, while on the S-exposed side, it decreased to $79.7^{\circ}$. The relaxation in the less stable $(10 \overline{1} 0) b$ edge was more pronounced. The S-Mo-S bond angles on the two edges were $90.7^{\circ}$ and $48.7^{\circ}$. The $(1 \overline{2} 10)$ edge relaxed in a similar manner.

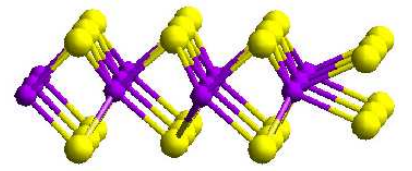

$(10 \overline{1} 0) \mathrm{b}$

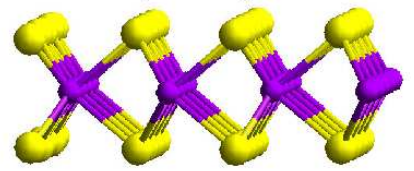

$(10 \overline{1} 0) \mathrm{a}$

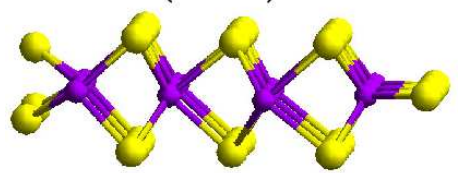

$(10 \overline{1} x)$

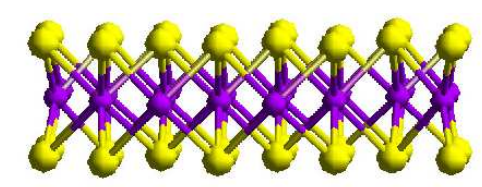

$(1 \overline{2} 10)$

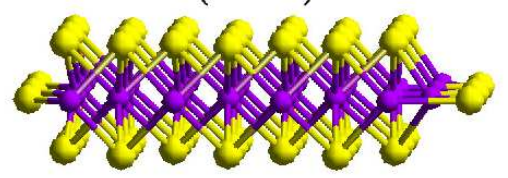

$(1 \overline{2} 1 x)$

Figure 3: Side views along the relaxed $\mathrm{MoS}_{2}$ edges. Each figure shows one or more unit cells of a 4-MoS ${ }_{2}$-unit wide ribbon which repeats into and out of the page. The left and right sides of each view are the actual edges. 
Table 2: Relaxed energies of the various $\mathrm{MoS}_{2}$ edges, measured per unit cell of the ribbon. All calculations were done with $4 \times 2$ unit cells. The large relaxation energy of the $(10 \overline{1} x)$ edge is due to reconstruction of the $\mathrm{S}$ atoms along that edge.

\begin{tabular}{cccc}
\hline Edge & $\begin{array}{c}E_{\text {tot }}(\text { relaxed }) \\
(\mathrm{eV})\end{array}$ & $\begin{array}{c}E_{\text {edge }}(\text { relaxed }) \\
\left(\mathrm{eV} / \mathrm{MoS}_{2} \text { unit }\right)\end{array}$ & $\begin{array}{c}\Delta E_{\mathrm{r}} \\
\left(\mathrm{eV} / \mathrm{MoS}_{2} \text { unit }\right)\end{array}$ \\
\hline$(10 \overline{1} 0) b$ & -188292.69 & 2.99 & -1.31 \\
$(10 \overline{1} 0) a$ & -188295.88 & 2.19 & -0.06 \\
$(1 \overline{2} 10)$ & -188296.81 & 1.96 & -0.17 \\
$(1 \overline{2} 1 x)$ & -188298.48 & 1.54 & -0.89 \\
$(10 \overline{1} x)$ & -188298.83 & 1.45 & -1.28 \\
\hline
\end{tabular}



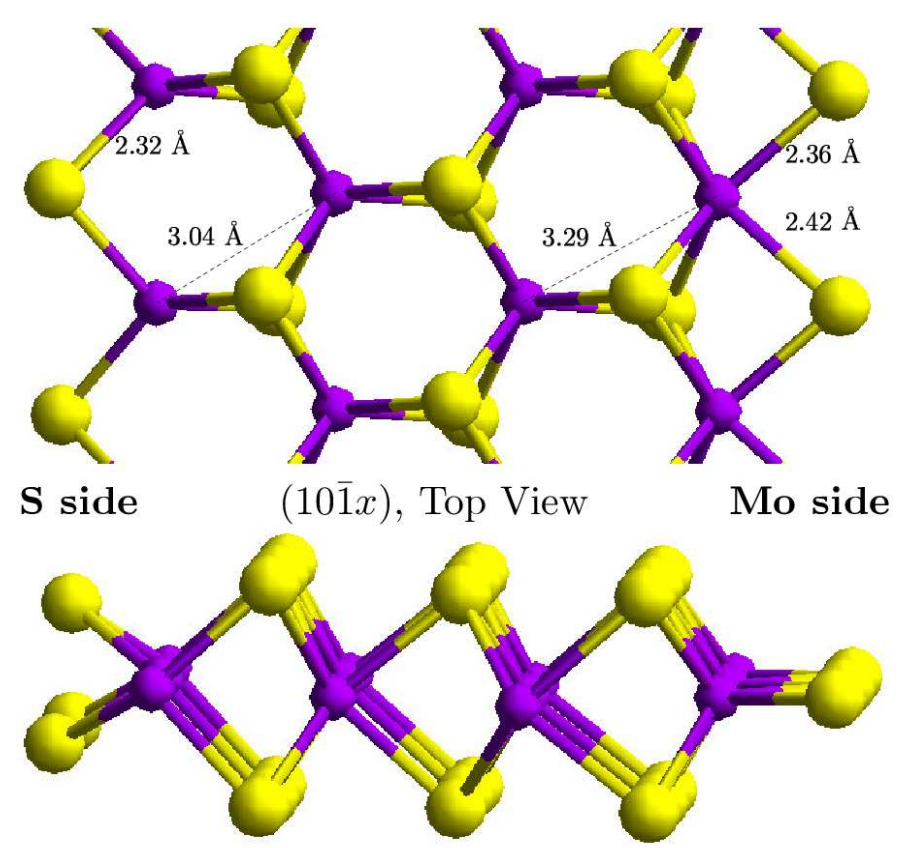

$(10 \overline{1} x)$, Side View

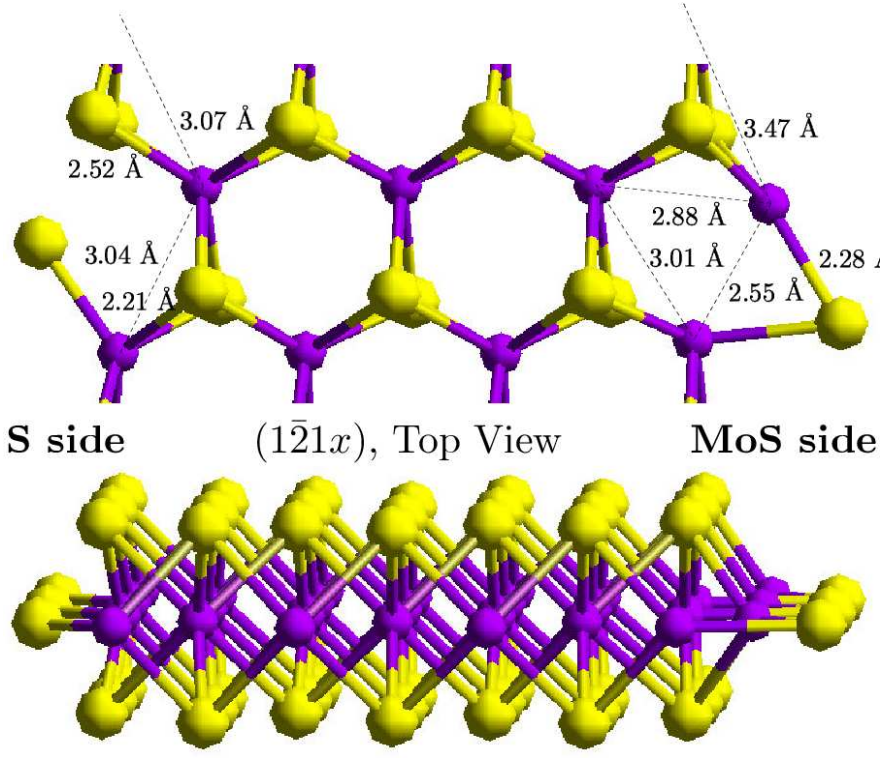

$(1 \overline{2} 1 x)$, Side View

Figure 4: The two most stable relaxed edges. Here the coordination of the edge Mo and S atoms is easily seen. Some Mo-Mo distances and Mo-S bond lengths near the edges are shown. Those distances which are not marked were within $0.1 \AA$ of the lengths from the optimized 2-D sheet, $d(\mathrm{Mo}-\mathrm{Mo})=3.16 \AA$ and $d(\mathrm{Mo}-\mathrm{S})=2.42 \AA$. The two complementary sides of each edge are labeled. 


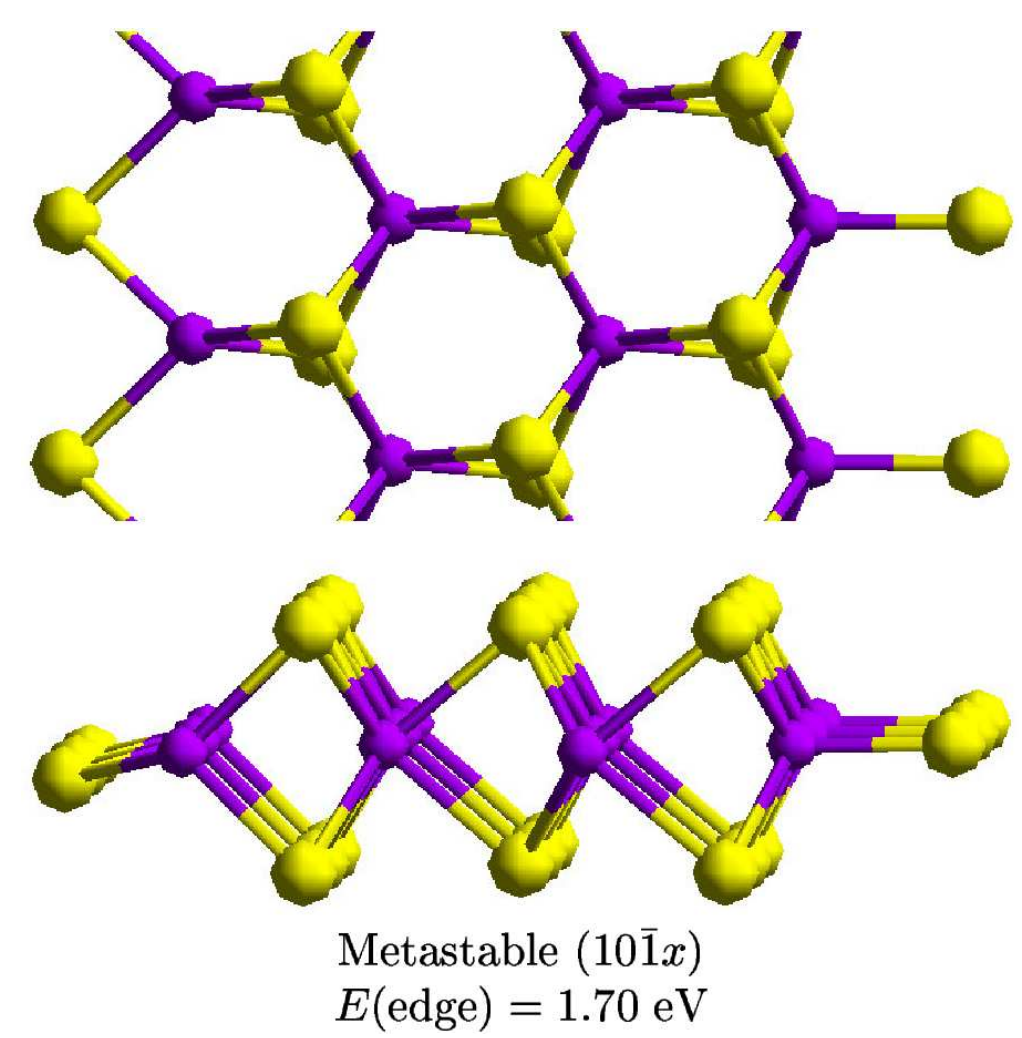

Figure 5: A metastable geometry for the $(10 \overline{1} x)$ edge. The local minimum was obtained from a direct optimization of the unrelaxed geometry, while the true minimum in Figure 4 was derived from a slightly disordered unrelaxed geometry. When the symmetry was broken, the optimization procedure found the more stable configuration, leading to an energy $0.25 \mathrm{eV}$ per edge $\mathrm{MoS}_{2}$ unit lower than this symmetric configuration. Note that the coordination of the Mo atoms on the right is only 5 and the $\mathrm{S}$ atoms on the left have not assumed their up-down configuration seen in Figure 3.

The behavior of the diagonally-cut edges was somewhat different. The $(1 \overline{2} 1 x)$ edge is simpler, so it is discussed first. The edge $\mathrm{S}$ atoms moved into the Mo-plane (Figures 3 and 4), keeping the same coordination and roughly the same Mo-S bond lengths. The Mo atoms moved slightly inward. The Mo-Mo distances and Mo-S bond lengths are given in Figure 4. On the MoS side, one Mo-Mo distance between unsaturated atoms decreased to $2.55 \AA$. These atoms had become almost bonded.

In the $(10 \overline{1} x)$ edge, something unique happened. Direct optimization of the bulkderived coordinates usually yielded a local minimum of energy at the geometry shown in Figure 5. The relaxation of the $\mathrm{S}$ atoms closely resembled that described below for the $\left(\mathrm{MoS}_{2}\right)_{7}$ cluster. In this periodic system, however, a deeper minimum was obtained with a slight shift of the edge $\mathrm{S}$ atoms on both sides. These edge $\mathrm{S}$ atoms were initially moved $0.1 \AA$ in random directions. This led to the fully relaxed geometry shown in Figure 4 . The Mo atoms on the Mo side changed coordination from a trigonal prismatic with one $\mathrm{S}$ 
vacancy to trigonal prismatic with the axis now parallel to the edge. The Mo atoms on the $\mathrm{S}$ side assumed a distorted tetrahedral configuration. The Mo-Mo distances are shown in Figure 4.

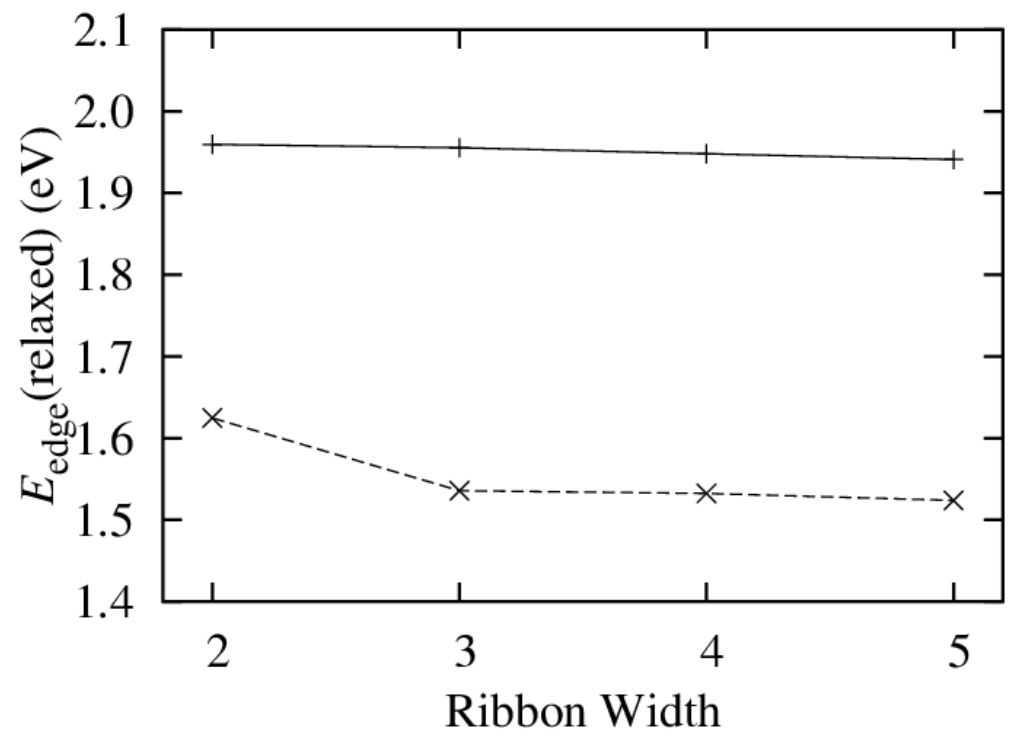

Figure 6: Test of the convergence of $E_{\text {edge }}$ for two representative edges as the ribbon becomes wider. The $4 \times 2$ ribbon was sufficient according to this result. The energies are shown for the (1210) (solid), and $(1 \overline{2} 1 x)$ (dashed) edges.

In this study, we also examined various ribbon widths to determine when edgeedge interactions were minimized. For the $(1 \overline{2} 10)$, $(10 \overline{1} x)$ (symmetric partial optimization), and $(1 \overline{2} 1 x)$ edges, $E_{\text {edge }}$ (relaxed) converged to within $9 \times 10^{-3} \mathrm{eV}$ by the $4 \times 2$ size (see Figure 6). The $3 \times 2$ ribbons were within $0.02 \mathrm{eV}$, while the energy of the $2 \times 2$ ribbons varied by up to $0.7 \mathrm{eV}$ from the larger sizes. The conclusion was that the $4 \times 2$ ribbon size is large enough to minimize the undesired interactions between edges, and this size ribbon was be used for most subsequent calculations.

The specific values of the "inclination index" $x$ in $(10 \overline{1} x)$ and $(1 \overline{2} 1 x)$ cannot be determined with certainty from a single-sheet model. However, because the bulk $\mathrm{MoS}_{2}$ structure includes the two 2-D sheets rotated with respect to each other by $180^{\circ}$, one can make a composite double-sheet edge structure by stacking two $180^{\circ}$-rotated single sheets as shown in Figure 7. Here the red lines yielded $x=3$ for $(10 \overline{1} x)$ and $x=4$ for $(1 \overline{2} 1 x)$ for stoichiometric $\mathrm{MoS}_{2}$ obtained by cuts of the ideal structure followed by relaxation. Another possible formation mechanism for the "inclined edges" is the migration of S atoms from one edge to the other, by $\mathrm{H}_{2} / \mathrm{H}_{2} \mathrm{~S}$. It has already been shown $[23,25]$ that the 
$(10 \overline{1} 0)$ edge will develop different structures due to adsorption and removal of $\mathrm{S}$ atoms in an $\mathrm{H}_{2} / \mathrm{H}_{2} \mathrm{~S}$ atmosphere. In a pure $\mathrm{H}_{2}$ atmosphere, $50 \% \mathrm{~S}$ coverage develops on both edges, which is equivalent to our $(10 \overline{1} x)$ edge.

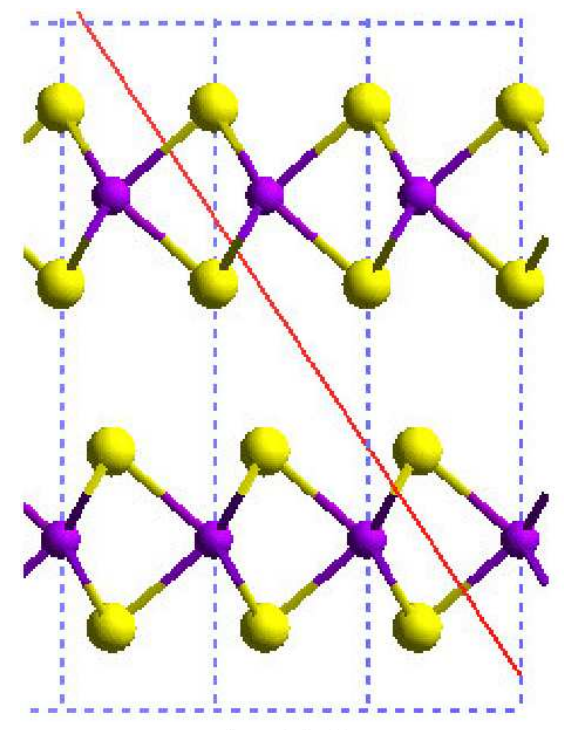

$(10 \overline{1} 3)$

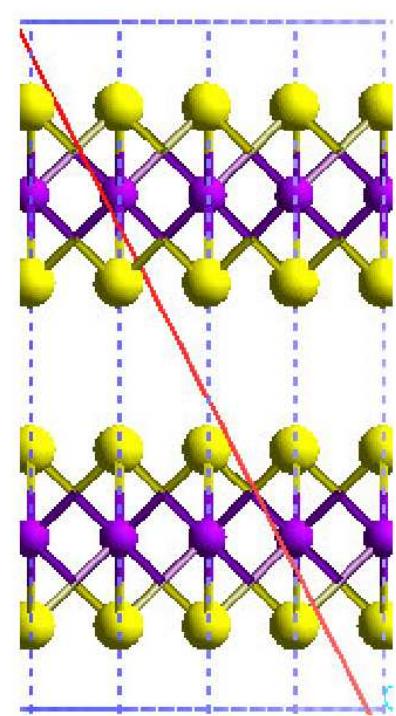

$(1 \overline{2} 14)$

Figure 7: Possible cleavage planes which would form "inclined edges" from a single $\mathrm{MoS}_{2}$ crystal.

\section{Electronic Structure of Edges}

The edge defect in the $\mathrm{MoS}_{2}$ sheet dramatically changed the electronic structure. The valence- and conduction-band DOS for a 2-D $\mathrm{MoS}_{2}$ sheet and for the most stable edges, $(1 \overline{2} 1 x)$ and $(10 \overline{1} x)$, are depicted in Figure 8 . In the 2-D sheet, five peaks were found in the valence band, as in previous valence band X-Ray photoelectron spectroscopy measurements by Park, et al. [31]. The peak positions for both the experiment and present work were determined by a fit to a series of Gaussian functions. The results are compared in Table 3. The peak intensities are not compared because the Scofield cross section [32] for Mo atoms is higher than for S atoms [33], so those peaks which are of Mo character were emphasized in the experimental DOS.

The band structure of 2-D $\mathrm{MoS}_{2}$ is shown in Figure 9. In the $\mathrm{MoS}_{2}$ sheet, the HOMO appeared at the top of the valence band, while the LUMO was at the bottom of the conduction band. The band gap was $1.8 \mathrm{eV}$, and valence band width was calculated to be $5.8 \mathrm{eV}$. For comparison, the experimental band gap of bulk $\mathrm{MoS}_{2}$ is $1.29 \mathrm{eV}$ [34]. 


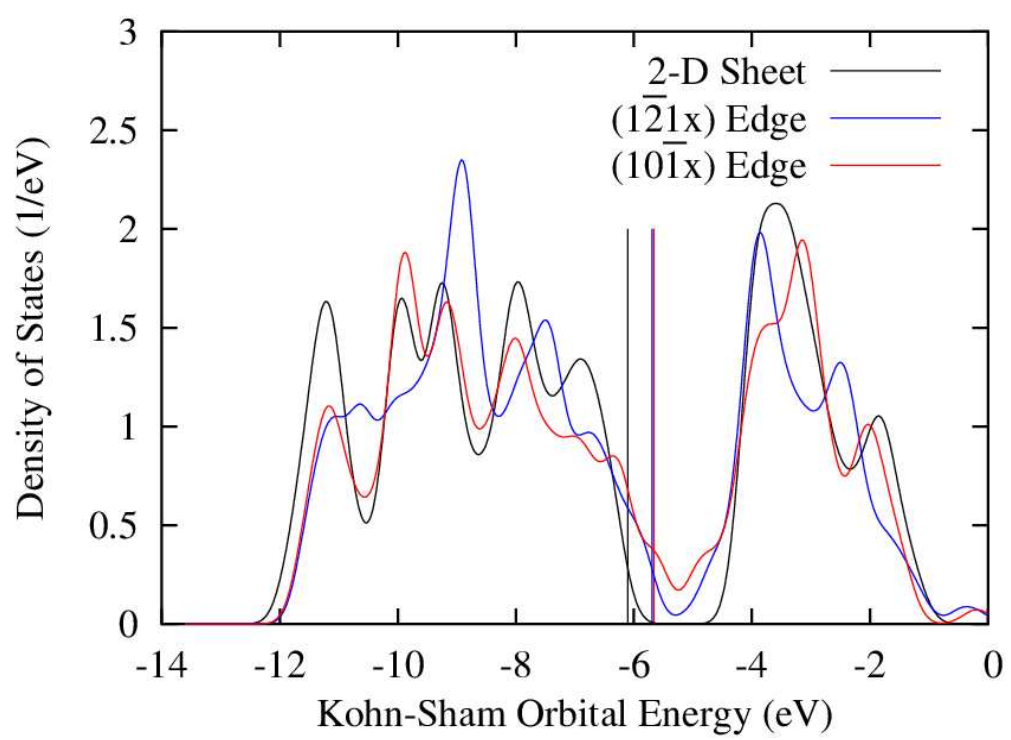

Figure 8: Calculated valence band and conduction band DOS of the 2-D MoS 2 sheet (black) and the most stable 1-D edges, (1 $\overline{2} 1 x)$ (blue) and $(10 \overline{1} x)$ (red). The DOS plots have been smoothed with a Gaussian having a FWHM of $0.5 \mathrm{eV}$. The HOMO energy in each case is marked with a vertical line.

Table 3: Valence-band peak locations (eV) from experiment [31] and the present calculations. The peaks are labeled by binding energy from the least to the most bound. The energies of the present calculation have been shifted so that the energy of peak A matches.

\begin{tabular}{ccc}
\hline Peak & $\begin{array}{c}\text { Park [31] } \\
\text { Experiment }\end{array}$ & $\begin{array}{c}\text { Present } \\
\text { Calculation }\end{array}$ \\
\hline A & -2.54 & -2.54 \\
B & -3.83 & -3.69 \\
C & -5.12 & -4.93 \\
D & -5.88 & -5.69 \\
E & -7.25 & -6.93 \\
\hline
\end{tabular}


Figure 10 shows the edge band structure. Because the model is one-dimensional, there is only one direction in $k$-space. For the $(1 \overline{2} 1 x)$ edge, this is the $\Gamma-\mathrm{M}$ direction, while for the $(10 \overline{1} x)$ edge, it is the $\Gamma-\mathrm{K}$ direction. Figure 11 shows the part of the band structure close to the Fermi level. The $(1 \overline{2} 1 x)$ edge is calculated to be semi-conducting, with the HOMO at the top of the valence band. There is a reduced, direct band gap of only $0.52 \mathrm{eV}$ at the $\Gamma$-point (Figure 10, Left).

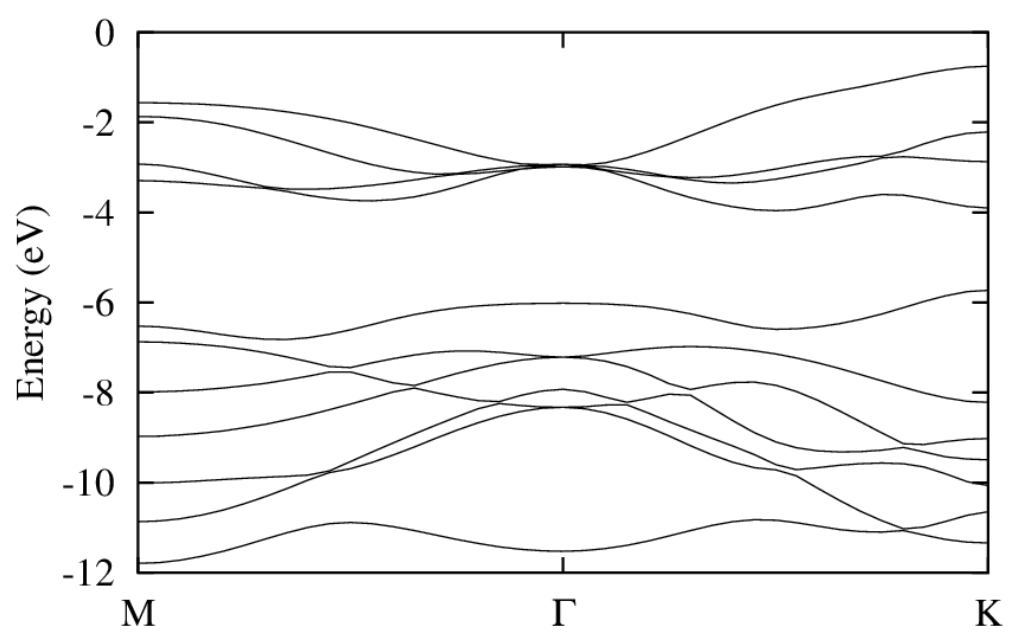

Figure 9: Upper valence band structure of the 2-D $\mathrm{MoS}_{2}$ sheet. 

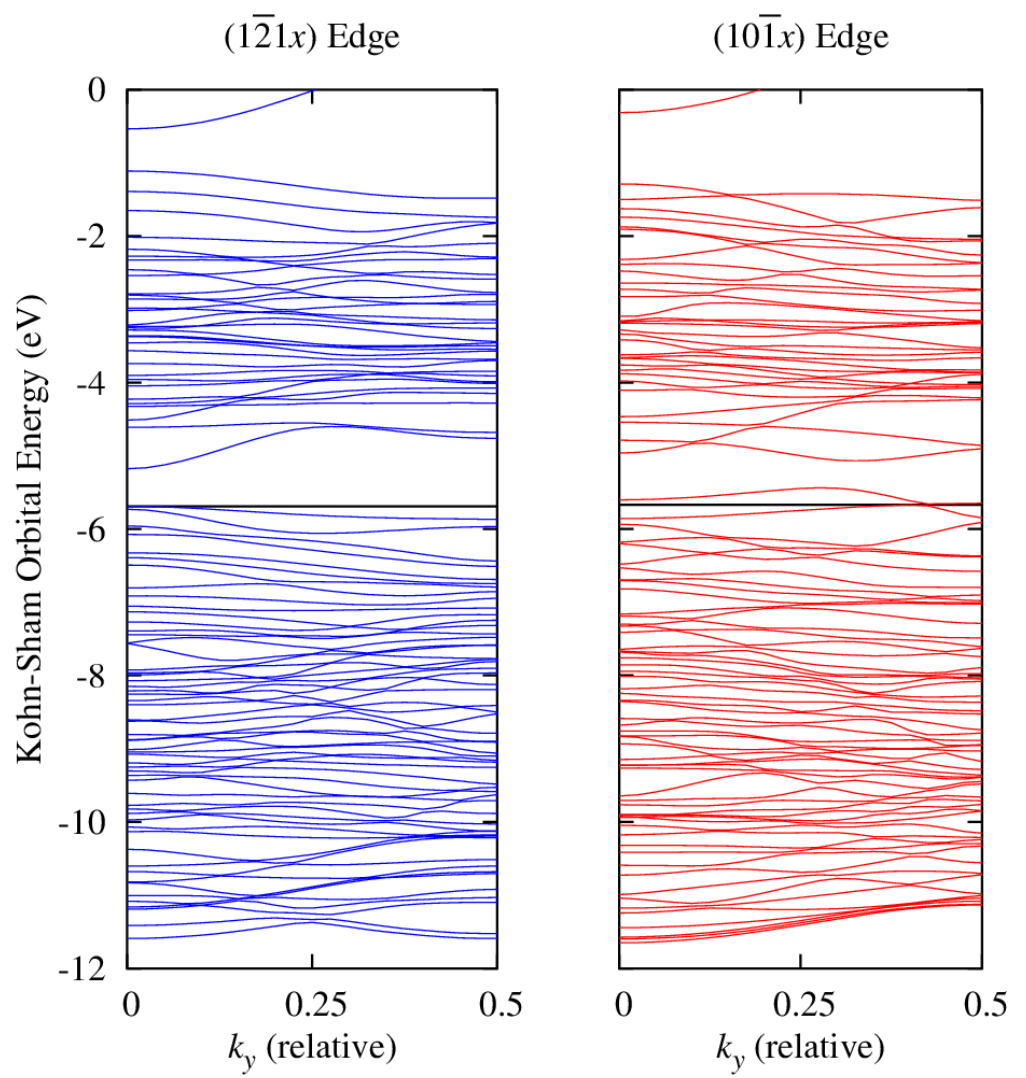

Figure 10: Valence band structure of the $(1 \overline{2} 1 x)$ and $(10 \overline{1} x)$ edge models of $\mathrm{MoS}_{2}$. The HOMO energies are marked with black lines. The HOMO of the $(10 \overline{1} x)$ edge was below the top of the valence band. Here $k_{y}$ is in units of $2 \pi / a$, where $a=6.32 \AA$ was the lattice constant for the $(10 \overline{1} x)$ edge and $a=5.473 \AA$ for the $(1 \overline{2} 1 x)$ edge. 
The HOMO of the $(10 \overline{1} x)$ edge, however, appeared $\sim 0.1 \mathrm{eV}$ below the top of the valence band (Figure 10, Right), leading to a Fermi edge, indicating a metallic character, in agreement with previous work [24,35]. There was one valence state just above the Fermi level.
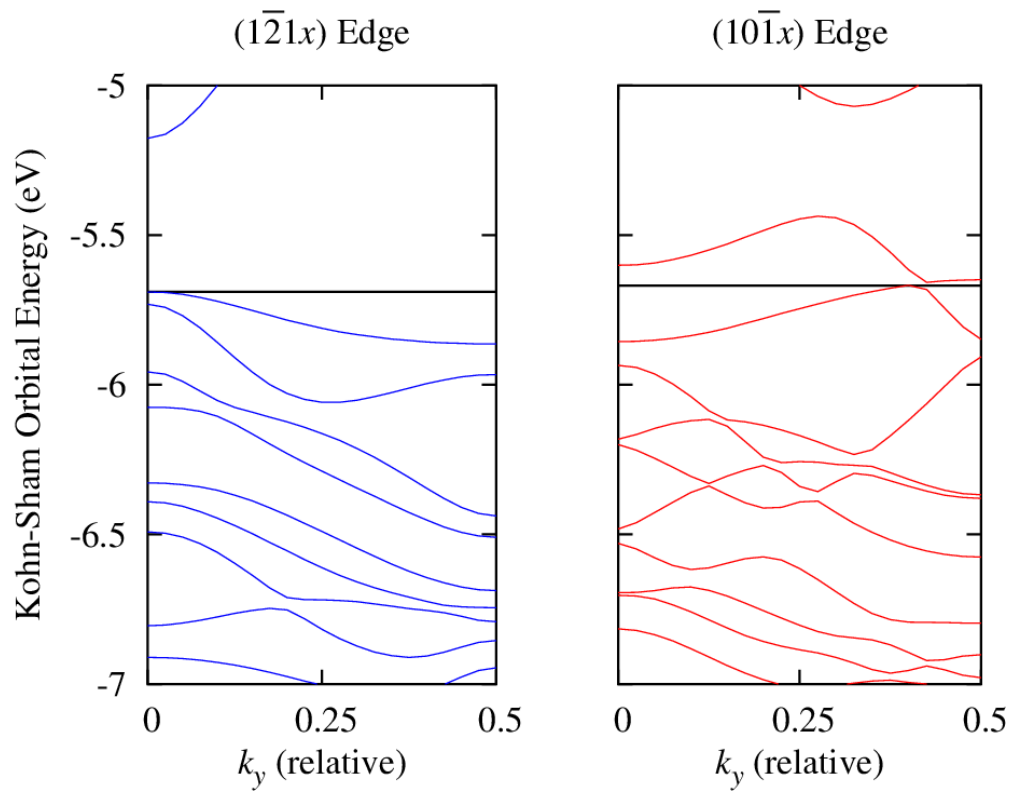

Figure 11: Upper valence band structure of the $(1 \overline{2} 1 x)$ and $(10 \overline{1} x)$ edges of $\mathrm{MoS}_{2}$. The Fermi energies are marked with black lines which separate the HOMO from the LUMO. An electron in the HOMO of the $(10 \overline{1} x)$ edge was found to have an effective mass of $1.9 m_{e}$. Here $k_{y}$ is in units of $2 \pi / a$, where $a=6.32 \AA$ was the lattice constant for the $(10 \overline{1} x)$ edge and $a=5.473 \AA$ for the $(1 \overline{2} 1 x)$ edge. 


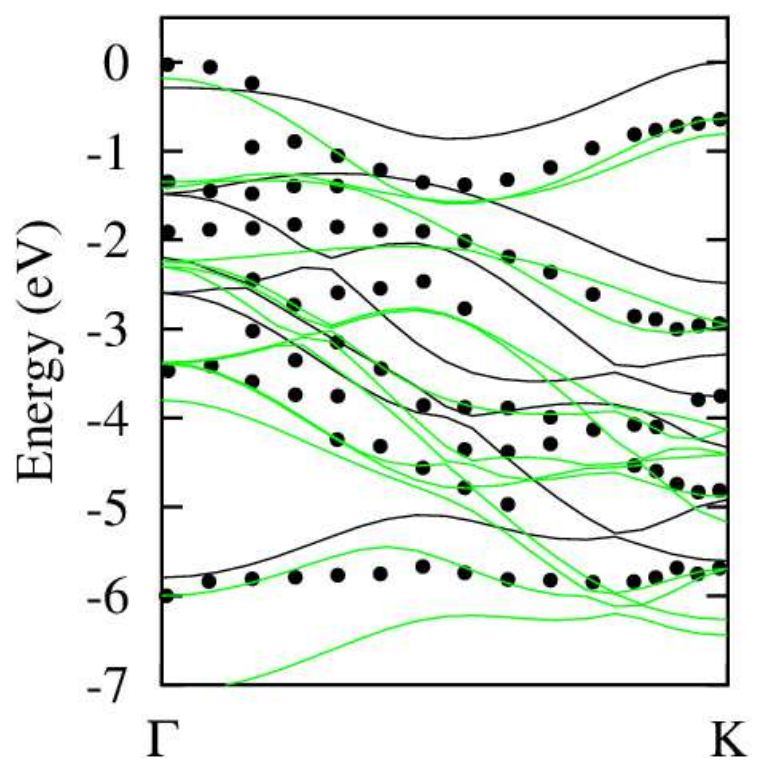

Figure 12: Experimentally derived band structure of $\mathrm{MoS}_{2}$ as determined by Böker [34] (circles) and the present calculation of the 2-D $\mathrm{MoS}_{2}$ sheet (black lines) and 3-D Bulk $\mathrm{MoS}_{2}$ (green lines).

The experimentally derived band structure of Böker [34] is compared with the 2-D sheet and the 3-D bulk $\mathrm{MoS}_{2}$ band structures in Figure 12. The experimental valence band width is about $6 \mathrm{eV}$, while the band gap is $1.29 \mathrm{eV}$. Our calculations of the 2-D $\mathrm{MoS}_{2}$ sheet found a valence band width of $5.8 \mathrm{eV}$ and a band gap of $1.8 \mathrm{eV}$. 3-D $\mathrm{MoS}_{2}$ had a calculated valence band width of $6.9 \mathrm{eV}$ and a band gap of $0.95 \mathrm{eV}$. The GGA functional is well-known to produce a poor band gap estimate, so this was expected.

A graphical representation of HOMO of the $(10 \overline{1} x)$ edge, a surface state, is shown in Figure 13. This state contained components of the $3 p$ orbitals of the edge sulfur atoms, the $4 \mathrm{~d}_{z^{2}}$ orbital of the Mo atoms nearest to the edge, and lesser contributions of other atoms. From the top view, we can see that this orbital was bonding both parallel and perpendicular to the edge. 

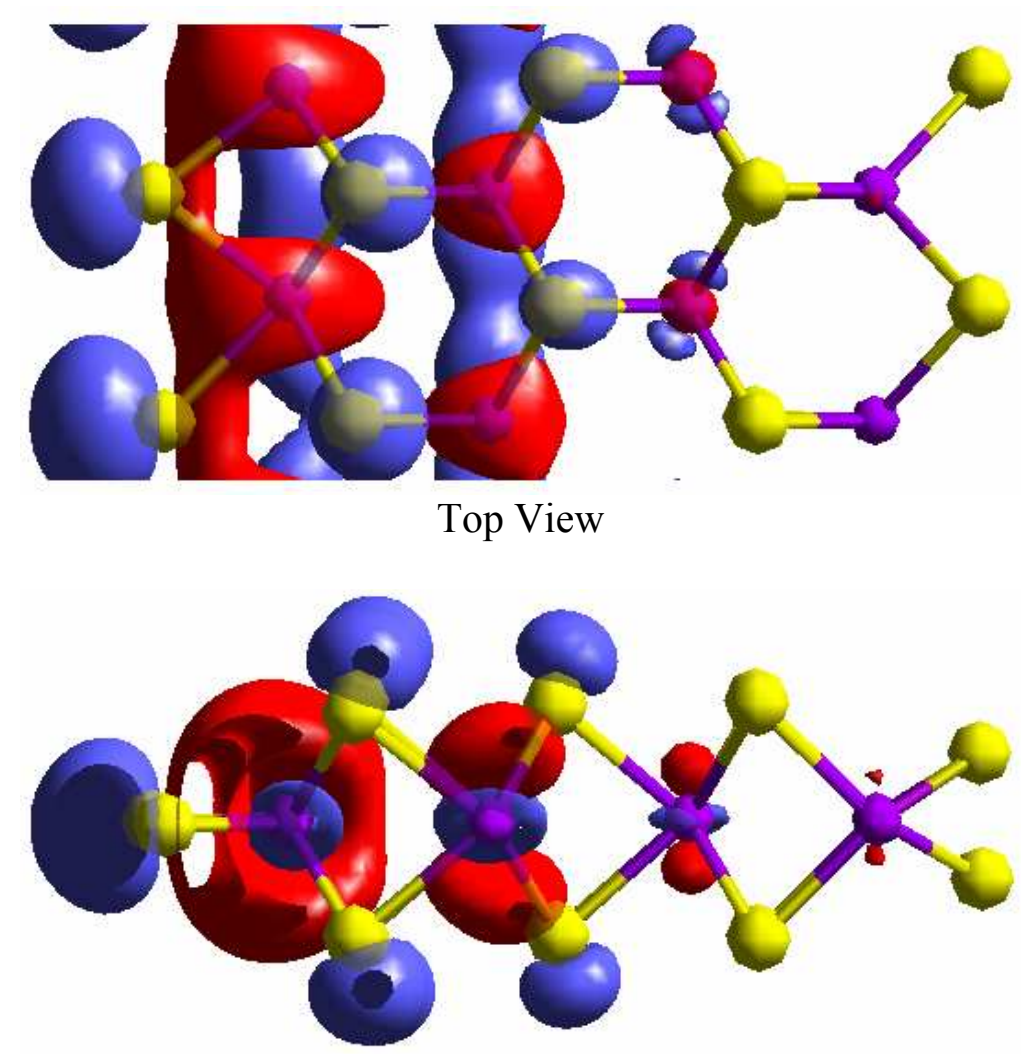

Side View

Figure 13: Surface state of the Mo-exposed side of the $(10 \overline{1} x)$ edge. This orbital is the HOMO at the $\Gamma$-point. Clearly seen are the edge sulfur $\mathrm{p}$ contributions, along with $\mathrm{d}$ orbitals of the edge Mo atoms aligned along the edge. The contributions of other atoms fall off as the distance from the edge increases. The orbital is cut off in the front and back by the walls of the repeating unit cell, leading to the white "hole" artifact seen in the ring of the Mo d orbital.

The bonding nature of this state in the propagation direction is reflected by its increasing orbital energy as the value of $k_{y}$ increases from zero at the $\Gamma$-point to higher values (Figure 10). The positive curvature of $\varepsilon_{\mathrm{HOMO}}$ dispersion means that the effective mass of an electron in that state is positive, and therefore the $(10 \overline{1} x)$ edge was determined to have an n-conducting character in the this direction when partially occupied due to a thermal excitation. The curvature yielded an effective mass of $1.9 \mathrm{~m}_{e}$, where $m_{e}$ is the mass of a free electron. This behavior is just opposite to that of the top of the 2-D valence band, which had a negative curvature of $\varepsilon_{\mathrm{HOMO}}$ and a negative effective mass of an electron, giving rise to p-conductivity with an effective mass of a hole of $4.1 m_{e}$. In the $(10 \overline{1} x)$ model, it was the HOMO-1 which resembled the $\varepsilon(k)$ curve of the bulk. 
Further into the Brillouin zone, the LUMO and HOMO of the $(10 \overline{1} x)$ ribbon became degenerate (or nearly degenerate) at $k \approx 0.4(2 \pi / a)$ and appeared to switch their occupancies at higher $k$ values up to $\pi / a$, the zone boundary, where the "new" HOMO again had a positive curvature of energy in $k$-space.

Even in this complicated system, the surface state behavior perpendicular to the edge followed that of surface states of a one-dimensional finite slab [36]. Specifically, the orbital was not concentrated solely on the surface itself; it extended into the subsurface layers. The form of the wavefunction in the 1-D case was a decaying exponential, where the rate of decay was determined by the perturbation of the atomic orbitals by the surface [36]. In this case, the depth of the surface state was about two layers, though different Mo d orbitals were involved on the surface and in the bulk.

\section{5. $\mathrm{MoS}_{2}$ Cluster Calculations}

Stoichiometric clusters of composition $\left(\mathrm{MoS}_{2}\right)_{n}, n=1,2,3$ and 7, were examined theoretically in order to assess the energies of formation and relaxation, and compare those with similar energy relationships calculated for edges in periodic systems, $c f$. above. The clusters were chosen to include structural motifs of a monomer $(n=1)$, dimer $(n=2)$, trimer $(n=3)$ and heptamer $(n=7)$, with sites exposing Mo atoms surrounded by various numbers of coordinated $\mathrm{S}$ atoms $(2,3,4,5$ and 6$)$ and sites exposing 1,2 and $3 \mathrm{~S}$ atoms. While several metastable geometries could be expected for each cluster size, the optimized structures in Figure 14 represent nuclei that can grow to the periodic layer by stepwise addition of $\mathrm{MoS}_{2}$ molecular units. The driving force for the crystal growth is the decrease of total energy per $\mathrm{MoS}_{2}$ formula with increasing cluster size. Furthermore, the clusters of this type are shown to have peripheral sites similar to those appearing at the edges of the periodic structures. The formal oxidation state of molybdenum in all clusters and periodic edge structures examined is $\mathrm{Mo}(\mathrm{IV}),[\mathrm{Kr}] 4 \mathrm{~d}^{2}$. This oxidation state is realized in the single $\mathrm{MoS}_{2}$ molecule [3] as well as in the perfect $\mathrm{MoS}_{2}$ crystal. Clusters with $n=2,3,7$ and $\mathrm{MoS}_{2}$ crystals with edge defects have reactive sites which will change the oxidation state of $\mathrm{Mo}(\mathrm{IV})$ upon adsorption of electron donor and acceptor molecules, an issue addressed is subsequent studies, wherein the reactivity of these sites toward hydrogen and alkali atoms (Part II), and CO (Parts III and IV) is examined. The relative stabilities of spin singlets and triplets in the $\left(\mathrm{MoS}_{2}\right)_{n}$ clusters were also investigated in order to determine the role of spin multiplicity when going from small to large clusters to solid $\mathrm{MoS}_{2}$.

The cluster structures were derived from the initial geometry of single-crystal $\mathrm{MoS}_{2}$ and were subsequently optimized as follows: for $n=1,2$, and 3, all Mo and S atoms were allowed to move to a minimum energy configuration, i.e. the $\mathrm{MoS}_{2}, \mathrm{Mo}_{2} \mathrm{~S}_{4}$, and $\mathrm{Mo}_{3} \mathrm{~S}_{6}$ clusters were fully optimized; for $n=7$, only peripheral Mo and $\mathrm{S}$ atoms were allowed to relax. Thus the $\mathrm{Mo}_{7} \mathrm{~S}_{14}$ cluster has an $\mathrm{Mo}_{3} \mathrm{~S}_{10}$ kernel of the ideal crystal structure with 4 peripheral Mo and 4 peripheral S atoms relaxed, to simulate the (10 $\overline{1} 0)$ and the $(10 \overline{1} x)$ edges examined above. The initial and the relaxed structures are shown in Figure 14. The largest, $\mathrm{Mo}_{7} \mathrm{~S}_{14}$, cluster indeed displays relaxation of peripheral atoms, 
e.g. that in Figure 14-c (left), similar to that of the edges in Figure 5 (10 $\overline{1} x$ ) (right). These relaxed structures are in fact very similar to those labeled $(10 \overline{1} 0)$ in ref. [26]. We reserve the inclination index $x$ in $(h k l x)$ for the movement of the edge atoms from unrelaxed to relaxed structures. Furthermore, the $\mathrm{Mo}_{7} \mathrm{~S}_{14}$ cluster is a borderline species between the high-spin smaller clusters and the zero-spin periodic $\mathrm{MoS}_{2}$ crystals, as will be seen below, and has intermediate electronic properties.
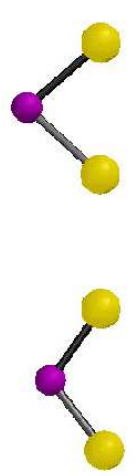

(a)
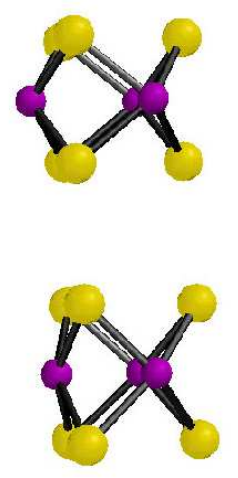

(b)
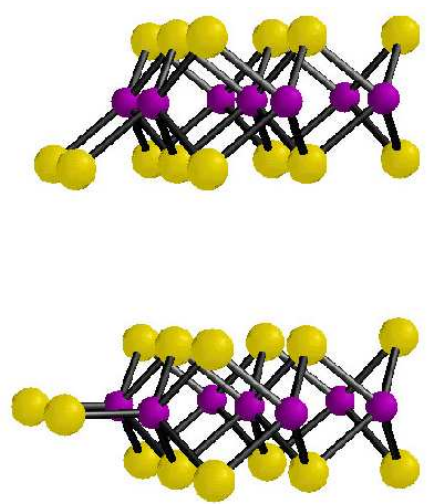

(c)

Figure 14: Initial (top) and relaxed (bottom) structures of $\left(\mathrm{MoS}_{2}\right)_{n}$ clusters, as viewed along the side resembling the $\mathrm{MoS}_{2}$ edges. The initial structures use the bond lengths and angles from the $\mathrm{MoS}_{2}$ crystal structure.
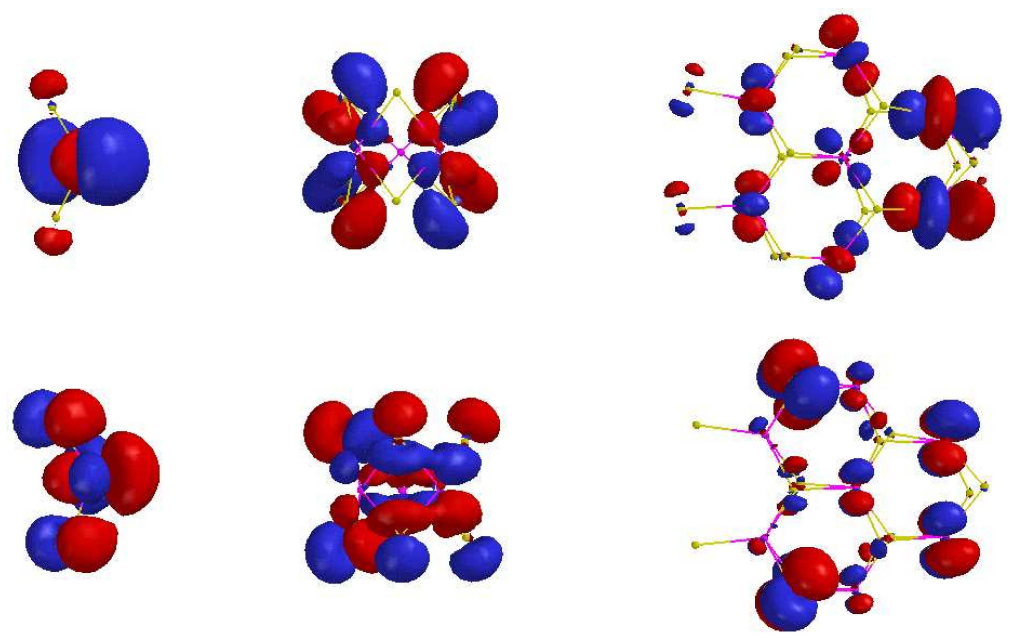

Figure 15: HOMO isosurfaces in singlet (top) and triplet (bottom) $\left(\mathrm{MoS}_{2}\right)_{n}$ clusters.

The structural relaxations are summarized as follows: The $\mathrm{MoS}_{2}$ monomer molecule is bent in both the spin singlet and triplet states, with Mo-S bonds shorter and 
S-Mo-S angles wider than in the crystal structure. The $\left(\mathrm{MoS}_{2}\right)_{2}$ dimer is highly asymmetric and its optimize structure shows no relation to the ideal crystal. The $\left(\mathrm{MoS}_{2}\right)_{3}$ trimer is highly symmetric and the exposed Mo atoms relax inward, the Mo-S bonds are longer, the S-Mo-S angles are slightly larger, and the Mo-Mo distances are significantly shorter than those in the ideal crystal structure. This attractive interaction of Mo atoms in small clusters has also emerged from CNDO/UHF calculations of Lobos [27].

Most remarkable is the similarity between structural relaxation of the peripheral atoms in the $\left(\mathrm{MoS}_{2}\right)_{7}$ heptamer and the edge relaxations presented above. On the "perpendicular cut" simulating the $(10 \overline{1} 0)$ edge (Figure 14-c, right), the relaxation is relatively small, while on the 'inclined cut' that simulates the (10 $1 x$ ) edge (Figure 14-c, left), the relaxation is very large primarily due to the movement of the two outer $\mathrm{S}$ atoms from the sulfur plane in the ideal crystal to the plane of the intra-sheet Mo atoms. This latter feature closely resembles the relaxation of the periodic $(10 \overline{1} x)$ edge (Figure 5 above) and has the net effect of changing the local structure of the exposed pentacoordinated Mo atoms from a trigonal prism with one sulfur vacancy to a square pyramidal site with buried Mo. 
Table 4: Energies of formation $\Delta E_{\mathrm{f}}$, relaxation $\Delta E_{\mathrm{r}}$, and of the triplet and singlet states in the $\left(\mathrm{MoS}_{2}\right)_{n}$ clusters. All energies are expressed in $\mathrm{eV} / \mathrm{MoS}_{2}$ unit.

\begin{tabular}{ccccc}
\hline Energy & $\mathrm{MoS}_{2}$ & $\mathrm{Mo}_{2} \mathrm{~S}_{4}$ & $\mathrm{Mo}_{3} \mathrm{~S}_{6}$ & $\mathrm{Mo}_{7} \mathrm{~S}_{14}$ \\
\hline$\Delta E_{\mathrm{f}}$ & 0 & -2.11 & -1.91 & -2.61 \\
$\Delta E_{\mathrm{r}}$ & -1.50 & - & -0.89 & -0.20 \\
$\Delta E_{\text {spin }}$ & 0.53 & 0.07 & 0.03 & 0.01 \\
$E_{\text {Triplet }}$ (relaxed) & -129924.60 & -129926.71 & -129926.52 & -129927.21 \\
$E_{\text {Singlet }}($ (relaxed $)$ & -129924.07 & -129926.64 & -129926.49 & -129927.20 \\
$E_{\text {Triplet }}($ unrelaxed $)$ & -129923.10 & - & -129925.63 & -129927.01 \\
$E_{\text {Singlet }}($ unrelaxed $)$ & -129922.46 & - & -129925.57 & -129927.00 \\
\hline
\end{tabular}

${ }^{a}$ The Mo7S14 cluster was partially optimized by allowing movement of only the Mo and $\mathrm{S}$ atoms on the $(10 \overline{1} x)$ and $(10 \overline{1} 0)$ surfaces.

${ }^{\mathrm{b}} \Delta E_{\mathrm{f}}=E\left(\mathrm{Mo}_{n} \mathrm{~S}_{2 n}\right)-E\left(\mathrm{MoS}_{2}\right)$ both being the relaxed ground states.

${ }^{\mathrm{c}} \Delta E_{\mathrm{r}}=E_{\text {Triplet }}($ relaxed $)-E_{\text {Triplet }}$ (unrelaxed). The unrelaxed structure is that of a mathematical cut from the $\mathrm{MoS}_{2}$ crystal lattice.

${ }^{\mathrm{d}} \Delta E_{\text {spin }}=E_{\text {Singlet }}($ relaxed $)-E_{\text {Triplet }}($ relaxed $)$.
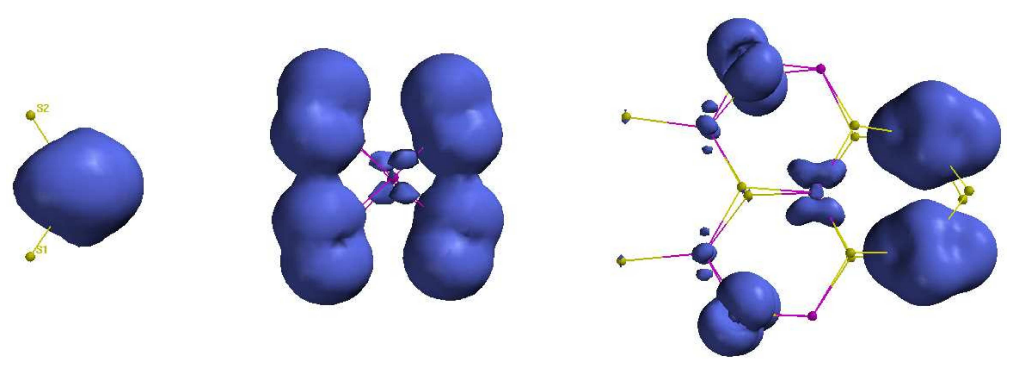

Figure 16: Spin density isosurfaces in triplet $\left(\mathrm{MoS}_{2}\right)_{n}$ clusters for $n=1,3$, and 7 .

The energy relationships in Table 4 are summarized as follows: (1) Large stabilization is gained upon the cluster growth, from $-2.11 \mathrm{eV} / \mathrm{MoS}_{2}$ unit accompanying the formation of $\left(\mathrm{MoS}_{2}\right)_{2}$ from the $\mathrm{MoS}_{2}$ molecule to $-0.70 \mathrm{eV} / \mathrm{MoS}_{2}$ unit going from the trimer to the heptamer, all optimized for the spin triplets; (2) The relaxation energies from the unrelaxed to the relaxed structures are also large, $-0.89 \mathrm{eV} / \mathrm{MoS}_{2}$ unit in the trimer, primarily due to the Mo-Mo interaction, and $-0.20 \mathrm{eV} / \mathrm{MoS}_{2}$ unit in the heptamer, primarily due to the reconstruction of the penta-coordinated Mo site; and (3) The spin 
triplet is more stable than the singlet in the monomer and trimer, and is nearly equally stable as the singlet in the heptamer. The $\left(\mathrm{MoS}_{2}\right)_{\infty}$ crystal is diamagnetic [37] and its spin singlet is most stable, as correctly accounted for by the DFT theory in earlier studies [2] as well as in the present work.

The HOMOs and the net spin density distributions in the triplet state of the clusters are shown in Figures 14 and 15. The HOMO has an Mo $4 \mathrm{~d}_{z^{2}}$ character in the singlet $\mathrm{MoS}_{2}$ molecule, a mixed Mo4d-S2p character in $\left(\mathrm{MoS}_{2}\right)_{3}$, and again Mo4d character in the largest cluster, $\left(\mathrm{MoS}_{2}\right)_{7}$, all antibonding or non-bonding atomic-like orbitals. These results are to be compared with the well-documented $M o 4 d_{z^{2}}$ antibonding character of the top of the valence band in the $\mathrm{MoS}_{2}$ crystal $[31,37]$. The HOMO in the triplet clusters resembles, as expected, the LUMO in the singlets. However, the triplet spin density, which represents a combined density of the hole and the electron forming the triplet (or 'exciton'), results from spin coupling due to exchange interaction which stabilizes the electronic high-spin state in highly correlated systems. The net effect is an increased delocalization of spin density from Mo to S with increasing cluster size and a simultaneously decreasing exchange-stabilization of the triplets. At the same time, it is predicted that very small $\mathrm{MoS}_{2}$ clusters will be paramagnetic and detectable by ESR or magnetic moment measurements.

\section{Cluster Calculations of $\mathrm{MoS}_{2}$ with Hydrogen}

To begin our study, we examined the simple $\mathrm{MoS}_{2}$ and $\mathrm{NbS}_{2}$ monomers. Hydrogen is bonded more weakly with increasing size of clusters, so these molecules show the strongest hydrogen attraction. The general theory of molecular hydrogen bonding to metals was recently reviewed by Kubas [38]. In his theory, Kubas categorized the $\mathrm{H}_{2}-$ metal bond as an $\eta^{2}-\mathrm{H}_{2}$ complex when the $\mathrm{H}-\mathrm{H}$ distance is small $(<0.9 \AA)$, an elongated $\mathrm{H}_{2}$ complex for intermediate $\mathrm{H}-\mathrm{H}$ distances, or a true dihydride only when the $\mathrm{H}-\mathrm{H}$ distance is larger $(>1.6 \AA)$. The present DFT calculations revealed both dihydride and $\eta^{2}-\mathrm{H}_{2}$ formation on the $\mathrm{MoS}_{2}$ molecule.

The reaction of $\mathrm{H}_{2}$ with the $\mathrm{MoS}_{2}$ monomer was found to be exothermic and released about $1.2 \mathrm{eV}$. The resultant $\mathrm{H}_{2}-\mathrm{MoS}_{2}$ molecule (Figure 17-a) had the symmetry $C_{2 v}$ and a singlet spin state. The spin is somewhat surprising, as the $\mathrm{H}_{2}$ molecule is a singlet while the $\mathrm{MoS}_{2}$ monomer was found to be most stable in the triplet state $[1,3]$. The $\mathrm{H}-\mathrm{H}$ distance was $3.08 \AA$ and the $\mathrm{Mo}-\mathrm{H}$ distances were $1.72 \AA$, consistent with a dihydride configuration. The $\mathrm{H}-\mathrm{Mo}-\mathrm{H}$ bond angle was $128^{\circ}$. 


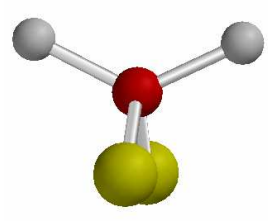

(a)

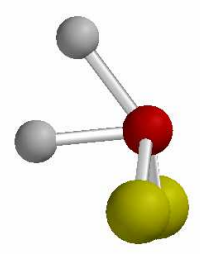

(b)

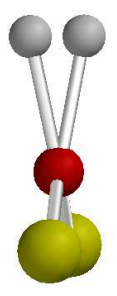

(c)

Figure 17: Stable and metastable geometries of $\mathrm{H}_{2}-\mathrm{MoS}_{2}$. Structure (a) is the ground state singlet dihydride, (b) is a metastable "tilted" configuration with an energy $0.3 \mathrm{eV}$ above the ground state, and (c) is the lowest-energy triplet $\eta^{2}$ hydrogen configuration, with an energy $0.6 \mathrm{eV}$ above the ground state.

Table 5: Various properties for the most stable singlet and triplet geometries of the $\mathrm{H}_{2}-\mathrm{MoS}_{2}$ complex.

\begin{tabular}{|c|c|c|}
\hline Property & Singlet & Triplet \\
\hline Geometry & Dihydride & $\eta^{2}-\mathrm{H}_{2}$ \\
\hline$d(\mathrm{H}-\mathrm{H})$ & $3.08 \AA$ & $0.80 \AA$ \\
\hline$d(\mathrm{H}-\mathrm{Mo})$ & $1.72 \AA$ & $2.04 \AA$ \\
\hline$\Delta \mathrm{E}_{\mathrm{f}}{ }^{1}$ & $-1.17 \mathrm{eV}$ & $-0.52 \mathrm{eV}$ \\
\hline$\angle(\mathrm{H}-\mathrm{Mo}-\mathrm{H})$ & $127.9^{\circ}$ & $22.5^{\circ}$ \\
\hline
\end{tabular}

Two metastable configurations of $\mathrm{H}_{2}-\mathrm{MoS}_{2}$ have been found. One is a singlet state in which the $C_{2 v}$ symmetry is broken by the movement of the $\mathrm{H}$ atoms to one side (Figure 17-b). The $\mathrm{H}$ and Mo atoms do lie on a mirror plane, so the overall symmetry is $C_{\mathrm{s}}$ or $C_{1 h}$. The energy of this state is $0.3 \mathrm{eV}$ higher than the minimum, the $\mathrm{H}-\mathrm{H}$ distance is $1.65 \AA$, and the $\mathrm{H}-\mathrm{Mo}-\mathrm{H}$ bond angle is $59^{\circ}$.

The other metastable geometry is the triplet configuration shown in Figure 17-c. It maintains the $C_{2 v}$ symmetry, but the $\mathrm{H}-\mathrm{H}$ distance is much smaller, only $0.80 \AA$. The triplet energy is about $0.6 \mathrm{eV}$ above the singlet minimum. The Mo-H distance is $2.04 \AA$, while the $\mathrm{H}-\mathrm{Mo}-\mathrm{H}$ bond angle is $22.5^{\circ}$.

Because this configuration has the same spin state as the separated $\mathrm{MoS}_{2}$ and $\mathrm{H}_{2}$ molecules, it is likely that the combination will first form this $\mathrm{H}_{2}-\mathrm{MoS}_{2}$ triplet, then later change into the ground state singlet configuration. How this occurs gives insight into the interaction between the Mo center and the $\mathrm{H}_{2}$ molecule that can help unravel the mechanism for dissociative hydrogen adsorption on the $\mathrm{MoS}_{2}$ edges found in the solid. 


\section{Coordinate Driving}

To calculate reaction rates and activation barriers between the described states, a continuous path through which the molecule can travel between the triplet and singlet configurations must be found. For this goal, constrained optimizations are performed. The chosen constraint is the $\mathrm{H}-\mathrm{Mo}-\mathrm{H}$ bond angle $\left(\theta_{\mathrm{H}}\right)$, as it is the most obvious visual difference between the ground state and triplet configurations. In the $\eta^{2}-\mathrm{H}_{2}$ geometry, as $\theta_{\mathrm{H}}$ is varied continuously from zero through the triplet value of $22.5^{\circ}$, the molecule maintains the triplet electronic state and no activation barrier is present. The $\mathrm{H}_{2}$ molecule is separated from $\mathrm{MoS}_{2}$ when $\theta_{\mathrm{H}} \rightarrow 0$. The energy is plotted as a function of $\theta_{\mathrm{H}}$ in Figure 18.

As $\theta_{\mathrm{H}}$ was increased to the singlet value of $128^{\circ}$, the coordinate-driven calculation revealed the formation of the dihydride. At an angle of around $30^{\circ}$, the lowest energy state is a singlet. Because of the $C_{2 \mathrm{v}}$ symmetry and the symmetry of the half-occupied orbitals, this is actually a forbidden transition. However, given enough time and/or a break in the symmetry, the singlet state could be obtained. At this point, two possible paths were found. Along one path, the "tilted" configuration in Figure 17-b was an intermediate, while the other path maintained the $C_{2 v}$ symmetry.

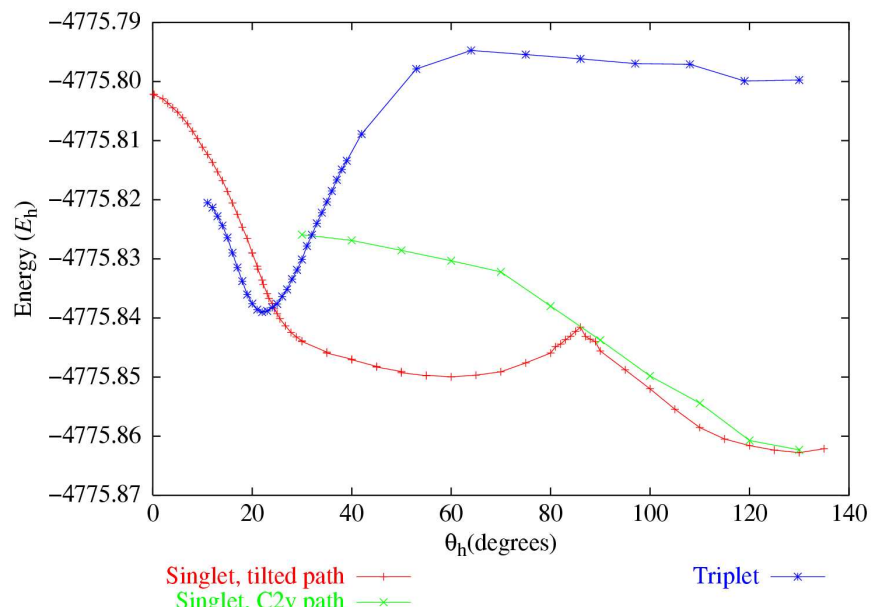

Figure 18: Constrained optimization of the energy of the $\mathrm{H}_{2} \mathrm{MoS}_{2}$ molecule as a function of the $\mathrm{H}-\mathrm{Mo}-\mathrm{H}$ bond angle $\theta_{\mathrm{H}}$. The hydrogen molecule is attracted to the exposed Mo atom in the $\mathrm{MoS}_{2}$ monomer (small $\theta_{\mathrm{H}}$ ) and could form a metastable $\eta^{2}-\mathrm{H}_{2}$ complex in a spin triplet. Overcoming a small activation energy leads to a stable configuration as a dihydride (large $\theta_{\mathrm{H}}$ ) in this coordinate-driven set of calculations. Easily seen are the metastable triplet state and a "tilted" singlet state, along with the ground singlet state. The upper singlet path maintains the $C_{2 v}$ symmetry, while the lower path breaks the symmetry to find the "tilted" configuration. 


\section{Orbital Analysis}

In his discussion of dihydride formation on metal complexes, Kubas [38] stressed the importance of overlap of the antibonding $\mathrm{H}_{2} \sigma^{*}$ orbital with orbitals in the metal complex. The overlap creates a molecular orbital which is a mixture of an occupied $d$ orbital in the metal with the $\sigma^{*}$ orbital of the $\mathrm{H}_{2}$. The resulting partial occupation of the $\sigma^{*}$ is termed backdonation. Figure 19 shows the orbitals with the strongest $\mathrm{H} \sigma^{*}$ contributions for the singlet and the triplet configurations, both of which have substantial contributions from the antibonding $\mathrm{H}_{2}$ orbital. In the singlet, this molecular orbital joins the $\mathrm{H}_{2} \sigma^{*}$ with the $\mathrm{S} p$ orbitals and is doubly occupied, as required by the zero total spin. In the triplet state, the $\sigma^{*}$-metal d orbital is only half occupied, while at the same time, the $\sigma$ orbital of the $\mathrm{H}_{2}$ is stabilized, leading to the $\eta^{2}-\mathrm{H}_{2}$ configuration of that complex.

The combination of $\mathrm{H}_{2}$ with $\mathrm{MoS}_{2}$ involves mainly a few "valence" orbitals. These are the $1 \mathrm{~s}$ orbitals of $\mathrm{H}$ (i.e. the $\sigma$ and $\sigma^{*}$ orbitals of $\mathrm{H}_{2}$ ), the $5 \mathrm{~d}$ orbitals of Mo, and the $3 p$ orbitals of $S$, a total of 13 orbitals of each spin. These levels are occupied by 14 electrons. Figure 20 shows the orbital energies of the separated $\mathrm{H}_{2}$ and $\mathrm{MoS}_{2}$ molecules, along with the triplet and singlet $\mathrm{H}_{2}-\mathrm{MoS}_{2}$ complexes. In the transition from separated molecules to the triplet state, the predominant change in the orbital energies was the stabilization of the $\mathrm{H}_{2} \sigma$ orbital. In particular, there was no change in the number of occupied orbitals of each symmetry. The singly-occupied B1 symmetry orbital number 38 of the $\mathrm{MoS}_{2}$ molecule came down slightly in energy, but not enough to cause the ground electronic state to be a singlet.

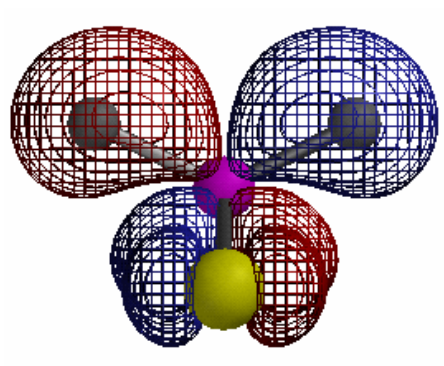

Singlet Dihydride

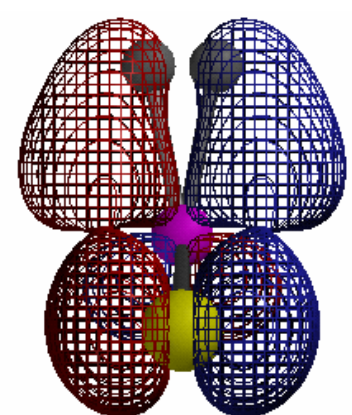

Triplet $\eta^{2}-\mathrm{H}_{2}$

Figure 19: Molecular orbitals of $\mathrm{H}_{2}-\mathrm{MoS}_{2}$ that have the highest $\mathrm{H}_{2} \quad \sigma^{*}$ character for the optimized singlet (left) and triplet (right) configurations. Both orbitals show interaction between the $\sigma^{*}$ and the Mo $\mathrm{d}_{\mathrm{yz}}$ orbital, but in the triplet case, this MO is only singly occupied. 


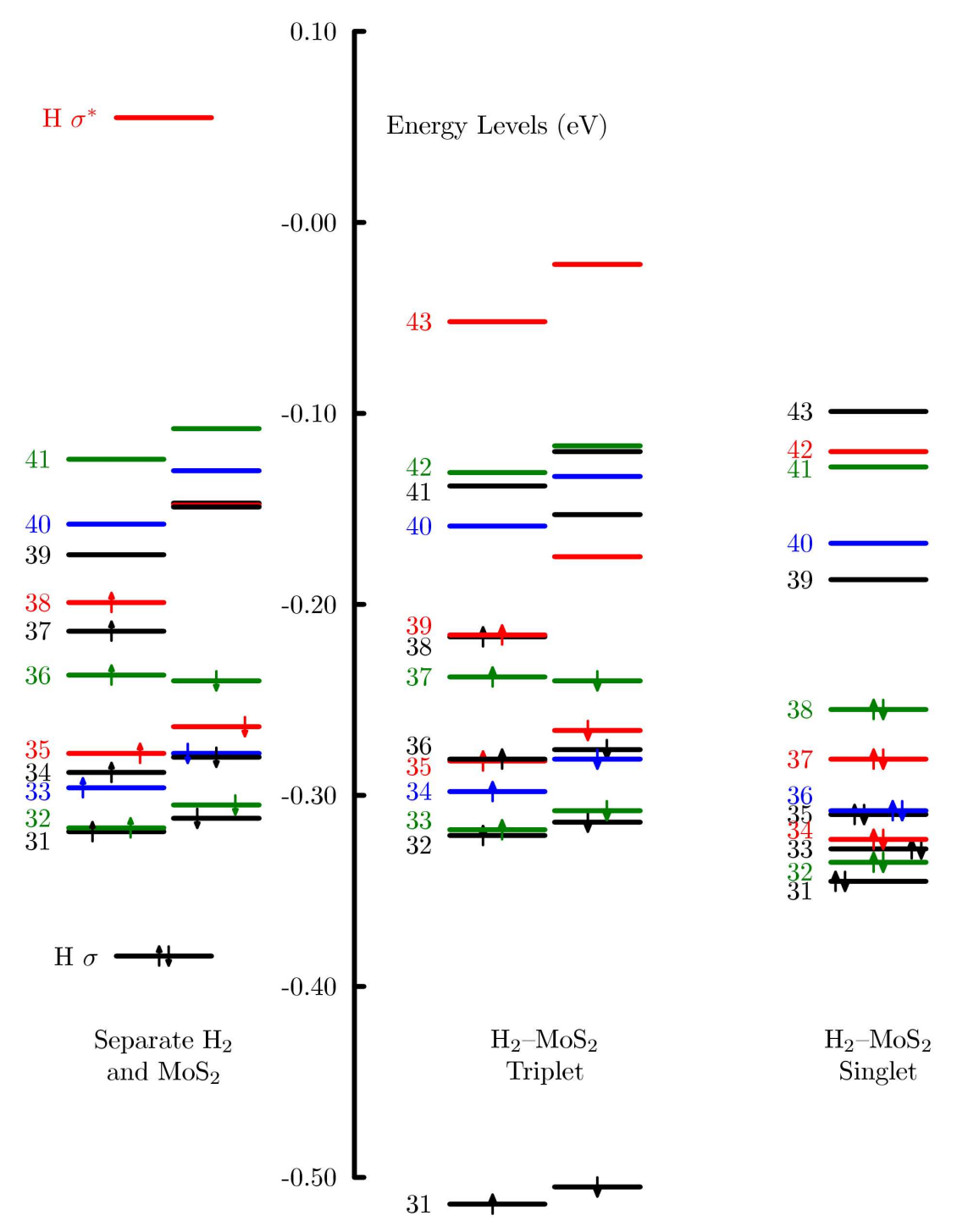

Figure 20: Energy levels of separated $\mathrm{H}_{2}$ and $\mathrm{MoS}_{2}$ monomer (left), the metastable triplet $\mathrm{H}_{2}-\mathrm{MoS}_{2}$ (center), and the ground state singlet $\mathrm{H}_{2}-\mathrm{MoS}_{2}$ (right). All geometries have the $C_{2 v}$ symmetry, and the orbitals are colored by their symmetries (black $=\mathrm{A} 1$; blue $=\mathrm{A} 2$; red $=\mathrm{B} 1$; green=B2). Between the triplet and singlet geometries, one A1 spin-up electron moves to the B1 spin-down orbital, a forbidden transition.

In the singlet state, there are several differences. With increased separation of the $\mathrm{H}$ atoms, the gap between the $\sigma$ and $\sigma^{*}$ orbitals was reduced. This caused the rise in the energy of the lowest valence orbital number 31. At the same time, the orbitals with $\sigma^{*}$ character (34 and 37 in Figure 20, right) became more stabilized. 
The question is how the transition from triplet to singlet occurs. Because of the change in the symmetries of the occupied orbitals, it is a forbidden transition. The coordinate-driven calculations of the intermediate states along the $C_{2 v}$ path show a sudden change in the multiplicity of the electronic ground state at an angle $\theta_{\mathrm{H}}$ of about $25^{\circ}$. The change of spin is a forbidden transition unless a spin-spin coupling provides a mechanism for switching from the triplet to the singlet state.

\section{Other Clusters}

Niobium disulfide $\left(\mathrm{NbS}_{2}\right)$ has also been studied because of structural similarity to $\mathrm{MoS}_{2}$ except that it has one less electron. Because of the odd number of electrons, the ground state of the $\mathrm{NbS}_{2}$ molecule is a spin doublet. There is a metastable dihydride $\mathrm{NbS}_{2}-\mathrm{H}_{2}$ complex, though the energy is about $0.4 \mathrm{eV}$ higher than the separate molecules. In this configuration, the $\mathrm{H}-\mathrm{H}$ distance is $3.14 \AA$, while the $\mathrm{Nb}-\mathrm{H}$ distance is $1.79 \AA$. The $\eta^{2}-\mathrm{H}_{2}-\mathrm{NbS}_{2}$ configuration is $0.16 \mathrm{eV}$ more stable than the dihydride. In this geometry, the $\mathrm{H}-\mathrm{H}$ distance is $0.762 \AA$, only slightly stretched from the equilibrium distance of $0.746 \AA$ in the free hydrogen molecule [39].

The effect of potassium doping: The presence of potassium dramatically alters the hydrogen adsorption properties of both the $\mathrm{MoS}_{2}$ and $\mathrm{NbS}_{2}$ molecules. Relative to the central metal atom, the optimized position of the potassium atom was between the sulfur atoms, as shown in Figure 21. The $\mathrm{H}_{2}-\mathrm{MoS}_{2} \mathrm{~K}$ dihydride complex was $45.7 \mathrm{kcal} / \mathrm{mol}$ more stable than separate $\mathrm{H}_{2}$ and $\mathrm{MoS}_{2} \mathrm{~K}$. The $\mathrm{NbS}_{2} \mathrm{~K}$ complex adsorbed hydrogen into the dihydride releasing $56.9 \mathrm{kcal} / \mathrm{mol}$ of energy. The $\mathrm{H}-\mathrm{H}$ and $\mathrm{Nb}-\mathrm{H}$ distances were $3.13 \AA$ and $1.79 \AA$, respectively.

Alkali doping also reduced the activation energy for forming a dihydride starting from the $\eta^{2}-\mathrm{H}_{2}$ configuration. Driving of the $\mathrm{H}-\mathrm{H}$ distance from the dihydride distance of $3.1 \AA$ to the molecular $0.7 \AA$ displayed a barrier of only about $0.22 \mathrm{eV}$ with the presence of $\mathrm{K}$, versus $0.7 \mathrm{eV}$ without the doping (Figure 22).
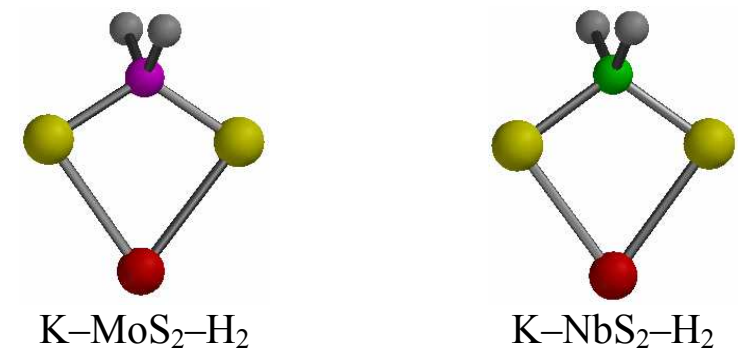

Figure 21: Geometries of the potassium-doped $\mathrm{MoS}_{2}$ and $\mathrm{NbS}_{2}$ monomers with $\mathrm{H}_{2}$ molecules attached to the transition metal. 

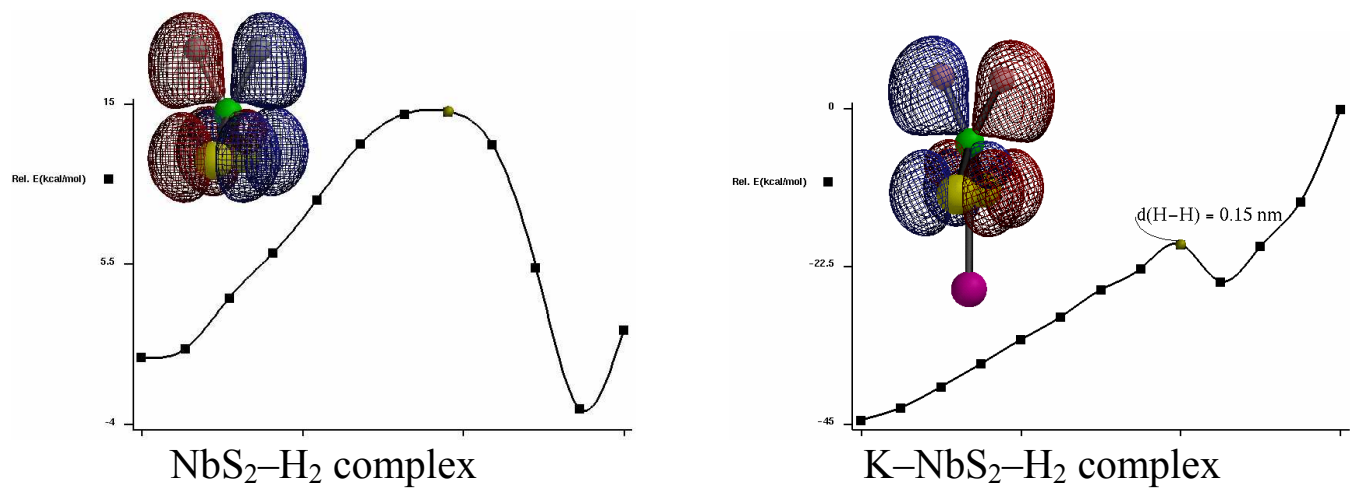

Figure 22: The addition of potassium to the $\mathrm{NbS}_{2}$ molecule significantly decreases both the activation energy and the final adsorption energy of $\mathrm{H}_{2}$ on the $\mathrm{NbS}_{2}$ monomer. The pictured orbital is the majority-spin HOMO at the top of the barrier, which is a combination of the $\sigma^{*}$ orbital of $\mathrm{H}_{2}$ and the $\mathrm{d}_{x z}$ orbital of $\mathrm{Nb}$, with some contribution from the $\mathrm{S}$ atoms.

\section{Hydrogen on $\mathrm{MoS}_{2}$ Clusters}

Calculations on larger clusters provided more information about the nature of adsorption sites and energies. As the cluster size increased, hydrogen was bonded more weakly. In our published paper [1] we presented a $\mathrm{Mo}_{7} \mathrm{~S}_{14}$ cluster with edges similar to those found in solid $\mathrm{MoS}_{2}$. The edges with exposed Mo were like the Mo-exposed (10 $\overline{1} 0$ ) edge of the $\mathrm{MoS}_{2}$ solid, while the edge with single exposed $\mathrm{S}$ atoms resembled the Mo-exposed $(10 \overline{1} x)$ edge.

This $\mathrm{Mo}_{7} \mathrm{~S}_{14}$ cluster was investigated for hydrogen adsorption in multiple locations. The $\left(\mathrm{MoS}_{2}\right)_{7}+5 \mathrm{H}_{2}$ cluster is pictured in Figure 23. The overall energy for the reaction

$$
\left(\mathrm{MoS}_{2}\right)_{7}+5 \mathrm{H}_{2} \rightarrow \mathrm{H}_{10}\left(\mathrm{MoS}_{2}\right)_{7}
$$

was calculated to be $+3 \mathrm{kcal} / \mathrm{mol} \mathrm{H}$, slightly endothermic. On this cluster, both $\eta^{2}-\mathrm{H}_{2}$ and the dihydride form were observed, with $\mathrm{H}-\mathrm{H}$ distances of $0.82 \AA$ and $1.97 \AA$. Both of these resembled hydrogen adsorbed on the Mo side of the $(10 \overline{1} 0)$ edge.

The effect of potassium doping: In preparation for calculations on the periodic $\mathrm{MoS}_{2}$ edges, sites for potassium adsorption on the $\left(\mathrm{MoS}_{2}\right)_{7}$ cluster were examined. The most stable site was found above the trigonal hole symmetrically situated between the two $(10 \overline{1} 0)$ edges, shown in Figure 24. The adsorption energy of the $\mathrm{K}$ atom was 7.78 $\mathrm{eV}$. The three nearest neighbors were two $\mathrm{S}$ atoms at distances of $3.14 \AA$ and one $\mathrm{S}$ atom at $3.17 \AA$. When five hydrogen molecules were adsorbed on the edges as above, the reaction was exothermic releasing $0.13 \mathrm{eV}$. The effect of the $\mathrm{K}$ was to stabilize the nearby Mo dihydrides. 


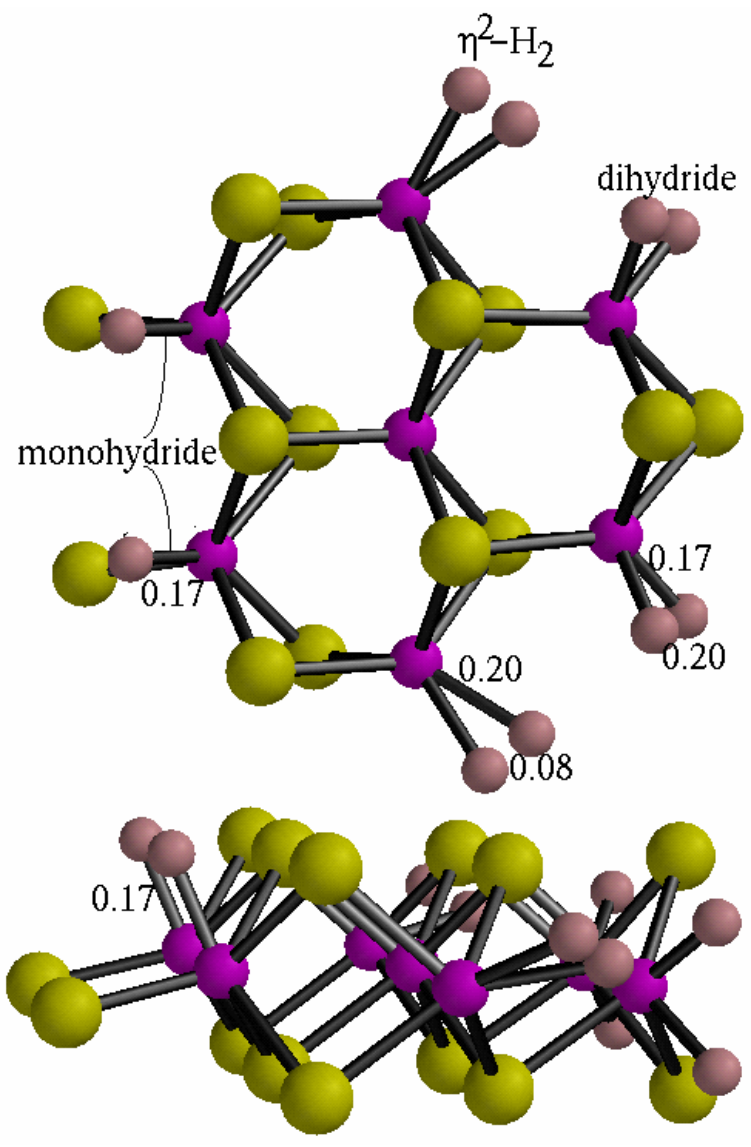

Figure 23: Geometry of hydrogen adsorption on the $\mathrm{Mo}_{7} \mathrm{~S}_{14}$ cluster. The left side of the cluster resembles the $(10 \overline{1} x)$ edge of the $\mathrm{MoS}_{2}$ crystal and has monohydrides. The top and bottom resemble the $(10 \overline{1} 0)$ edge and show both $\eta^{2}-\mathrm{H}_{2}$ and dihydride formation. Distances are in $\mathrm{nm}$. 

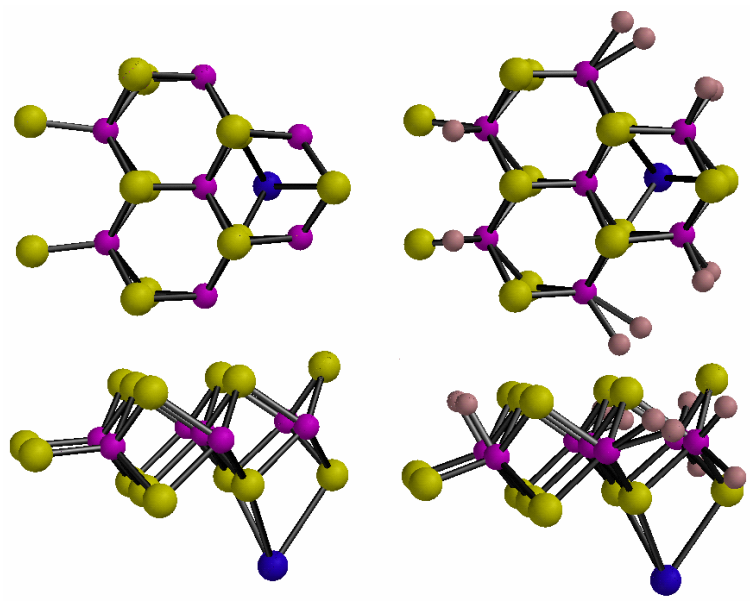

Figure 24: The most stable site for potassium adsorption on the $\mathrm{Mo}_{7} \mathrm{~S}_{14}$ cluster. The dihydrides on the Mo atoms nearest the $\mathrm{K}$ are stabilized by the addition of $\mathrm{K}$.

\section{Adsorption of $\mathrm{H}$ on Periodic $\mathrm{MoS}_{2}$}

The basal plane (0001) of $\mathrm{MoS}_{2}$ is quite stable and was found to be unreactive to hydrogen. The $\mathrm{H}$ atoms adsorbed releasing at most $0.59 \mathrm{eV}$ of energy in positions over the sulfur atoms or in the hollow site in the Mo-plane. The dissociation energy of the hydrogen molecule was $2.28 \mathrm{eV} /$ atom using the same theoretical model, so clearly dissociative adsorption of the hydrogen molecule on the basal plane would not occur.

Several adsorption sites for hydrogen atoms on the $(1 \overline{2} 1 x)$ and $(10 \overline{1} x)$ edges of $\mathrm{MoS}_{2}$ were tested. Both edges have two complementary sides. These were labeled in Figure 4 as the $\mathrm{S}$ and Mo sides on $(10 \overline{1} x)$, while they were denoted as the $\mathrm{S}$ and MoS sides on $(1 \overline{2} 1 x)$.

For each hydrogen adsorption location, the atoms in half of the unit cell were allowed to relax to a stable configuration. The edge geometries are shown in Figure 25. The calculations were performed with a $5 \times 2$ unit cell of $\mathrm{MoS}_{2}$ and a single hydrogen atom. The adsorption energies were calculated for the reaction

$$
\left(\mathrm{MoS}_{2}\right)_{n}+\mathrm{H} \rightarrow \mathrm{H}-\left(\mathrm{MoS}_{2}\right)_{n}
$$

so that the adsorption energy is defined as

$$
\Delta E=E\left(\mathrm{H}-\left(\mathrm{MoS}_{2}\right)_{n}\right)-\left[E\left(\left(\mathrm{MoS}_{2}\right)_{n}\right)+E(\mathrm{H})\right]
$$

With this definition, negative energies correspond to exothermic reactions. The final adsorption energies of each configuration are given in Table 6. 
The strongest binding of hydrogen was found on the $(1 \overline{2} 1 x)$ edge, $\mathrm{S}$ side, over the second-row $\mathrm{S}$ atom (c.f. Figure 25). With $\Delta E=-2.90 \mathrm{eV}$, there was more than enough energy to dissociate the $\mathrm{H}_{2}$ molecule. The interest here, however, is in adsorption on the metal centers. The strongest binding near Mo atoms was the Mo-bridge configuration on the $\mathrm{S}$ side of the $(10 \overline{1} x)$ edge. This configuration notably had the lowest vibrational frequency of only $1222.8 \mathrm{~cm}^{-1}$, while non-bridge positions had frequencies of at least $1700 \mathrm{~cm}^{-1}$.

The most exposed Mo atom of all of the edges was the Mo atom on the MoS side of the $(1 \overline{2} 1 x)$ edge. It is coordinated to only three $\mathrm{S}$ atoms in an approximately trigonal planar configuration. The expectation that this atom is especially reactive is tempered by the low binding energy of the $\mathrm{H}$ atom, only $-2.14 \mathrm{eV}$. The Mo atom on the opposite, $\mathrm{S}$ side of $(1 \overline{2} 1 x)$ was found to have a higher affinity for the $\mathrm{H}$ atom, with a binding energy of $-2.24 \mathrm{eV}$. The vibrational frequencies of the $\mathrm{H}$ atom at the two sites were fairly close, $1862.8 \mathrm{~cm}^{-1}$ on the $\mathrm{S}$ side and $1862.4 \mathrm{~cm}^{-1}$ on the MoS side.

Previous work by Cristol et al.[25,28] examined hydrogen adsorption on various $\mathrm{MoS}_{2}$ edges with DFT using a plane-wave pseudopotential basis set. On the $(10 \overline{1} x)$ edge (labeled " $[X-3]$ " for the Mo side and "[3-X]" for the S side in [25]), Cristol et al. found a minimum dissociative adsorption energy of $0.01 \mathrm{eV}$ on the $\mathrm{S}$ side of this edge and $0.27 \mathrm{eV}$ on the Mo side (both endothermic). The present calculations show exothermic binding of the $\mathrm{H}$ atoms on both edges. Using the adsorption energy for atomic hydrogen and the binding energy of the $\mathrm{H}_{2}$ molecule, the energy for the reaction:

$$
\mathrm{H}_{2}+\left(\mathrm{MoS}_{2}\right)_{n} \rightarrow 2 \mathrm{H}-\left(\mathrm{MoS}_{2}\right)_{n}
$$

was calculated as $-0.80 \mathrm{eV}$ (exothermic) on the Mo side of the $(10 \overline{1} x)$ edge and $-0.34 \mathrm{eV}$ on the $\mathrm{S}$ side. Both sides were active edges for exothermic adsorption of molecular hydrogen. 


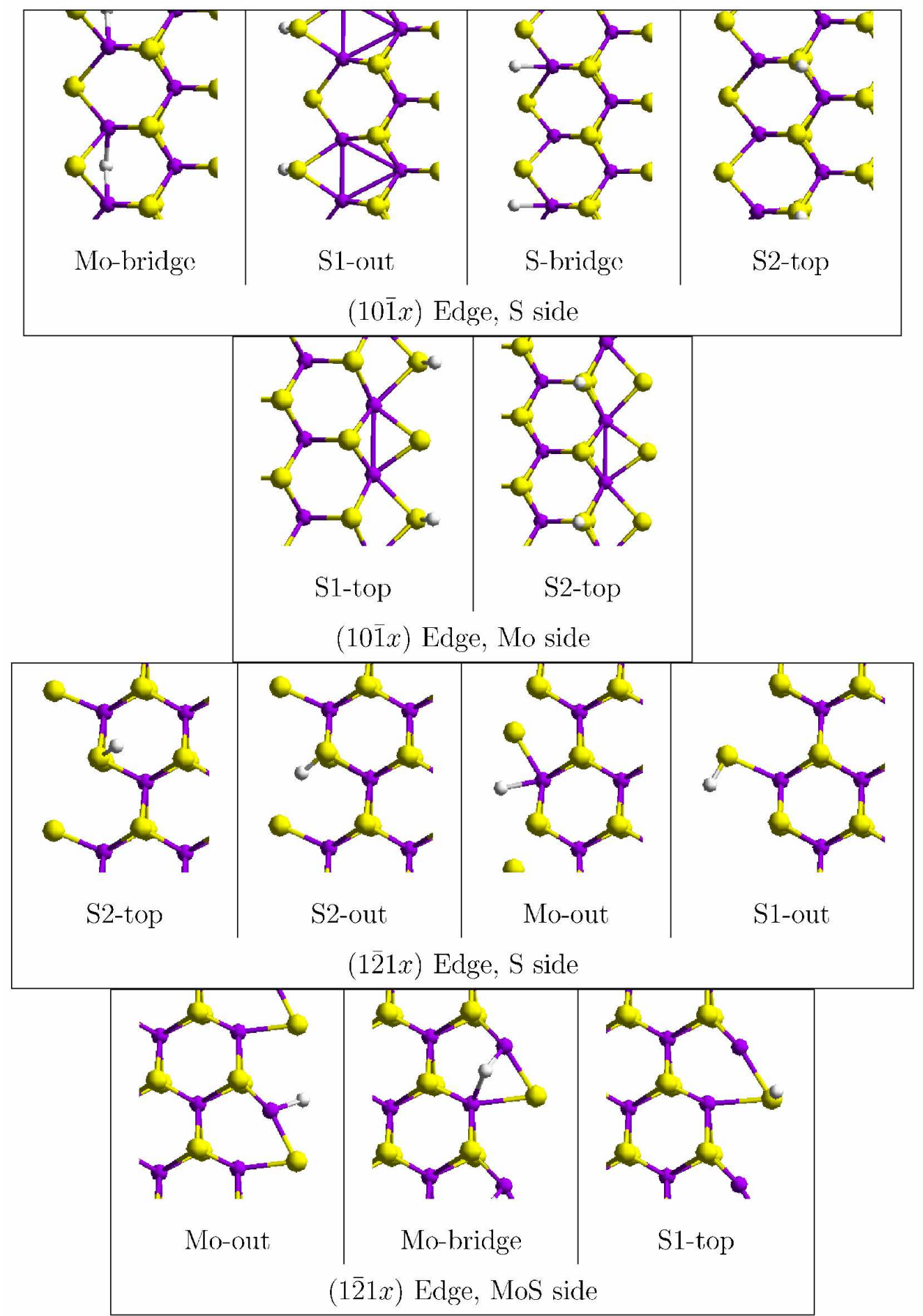

Figure 25: Geometries of adsorption locations for hydrogen atoms on the $(10 \overline{1} x)$ and $(1 \overline{2} 1 x)$ edges of $\mathrm{MoS}_{2}$. When two $\mathrm{H}$ atoms are seen, it is because of the repeating unit cell. 
Table 6: Adsorption energies of hydrogen atoms on $\mathrm{MoS}_{2}(10 \overline{1} x)$ and $(1 \overline{2} 1 x)$ edges. The binding energy of the $\mathrm{H}_{2}$ molecule is $-2.28 \mathrm{eV} /$ atom. The adsorption of hydrogen on the $\mathrm{MoS}_{2}$ basal plane is shown at the bottom. The labels of the sides are defined in Figure 4.

\begin{tabular}{ccccc}
\hline Edge & Side & Position & $\Delta \mathrm{E}$ & $v_{\max }$ \\
\hline$(10 \overline{1} x)$ & S side & Mo-bridge & $-2.45 \mathrm{eV}$ & $1222.8 \mathrm{~cm}^{-1}$ \\
& & S1-out & $-2.07 \mathrm{eV}$ & $2544.4 \mathrm{~cm}^{-1}$ \\
& & S-bridge & $-1.44 \mathrm{eV}$ & $1735.5 \mathrm{~cm}^{-1}$ \\
& & S2-top & $-1.42 \mathrm{eV}$ & $2455.0 \mathrm{~cm}^{-1}$ \\
\hline \multirow{4}{*}{$(1 \overline{2} 1 x)$} & Mo side & S1-top & $-2.68 \mathrm{eV}$ & $2588.6 \mathrm{~cm}^{-1}$ \\
& & S2-top & $-1.76 \mathrm{eV}$ & $2561.9 \mathrm{~cm}^{-1}$ \\
& & S2-top & $-2.90 \mathrm{eV}$ & $2550.2 \mathrm{~cm}^{-1}$ \\
& & S2-out & $-2.86 \mathrm{eV}$ & \\
& & S1-out & $-2.44 \mathrm{eV}$ & \\
& & Mo-out & $-1.82 \mathrm{eV}$ & $1862.8 \mathrm{~cm}^{-1}$ \\
& MoS side & Mo-out & $-2.14 \mathrm{eV}$ & $1862.4 \mathrm{~cm}^{-1}$ \\
& & Mo-bridge & $-1.82 \mathrm{eV}$ & $1570.8 \mathrm{~cm}^{-1}$ \\
& & S1-top & $-1.62 \mathrm{eV}$ & $2553.8 \mathrm{~cm}^{-1}$ \\
\hline
\end{tabular}

A limited number of $\mathrm{H}_{2}$ on $\mathrm{MoS}_{2}$ calculations were performed (Figure 26 and Table 7$)$. On the MoS side of the $(1 \overline{2} 1 x)$ edge, a dihydride and an $\eta^{2}-\mathrm{H}_{2}$ configuration were tested. The dihydride showed a metastable configuration with $\Delta \mathrm{E}=0.28 \mathrm{eV}$. Hydrogen was adsorbed exothermally in the $\eta^{2}-\mathrm{H}_{2}$ configuration with $\Delta \mathrm{E}=-0.52 \mathrm{eV}$. The vibrational frequencies in this geometry were $2999.2 \mathrm{~cm}^{-1}$ for $\mathrm{H}-\mathrm{H}$ stretching, $1483.9 \mathrm{~cm}^{-1}$ for the symmetric $\mathrm{H}_{2}-$ Mo stretching, and $838.4 \mathrm{~cm}^{-1}$ for the asymmetric $\mathrm{H}_{2}-$ Mo stretching. Here again, like the Mo-bridge configurations, the hydrogen atoms had a relatively low vibrational frequency.

On the Mo side of the $(10 \overline{1} x)$ edge, a configuration denoted $\mathrm{SH}-\mathrm{SH}$ was tested. This consisted of one $\mathrm{H}$ atom in the S1-top configuration with another on the bottom of the other edge $\mathrm{S}$ atom. The energy for dissociative adsorption here was $-0.06 \mathrm{eV}$. On the Mo side of the $(10 \overline{1} x)$ edge, a double Mo-bridge configuration was found to have an adsorption energy of $\Delta \mathrm{E}=-0.35 \mathrm{eV}$, very close to the $-0.34 \mathrm{eV}$ predicted by using the adsorption energy of an $\mathrm{H}$ atom. 


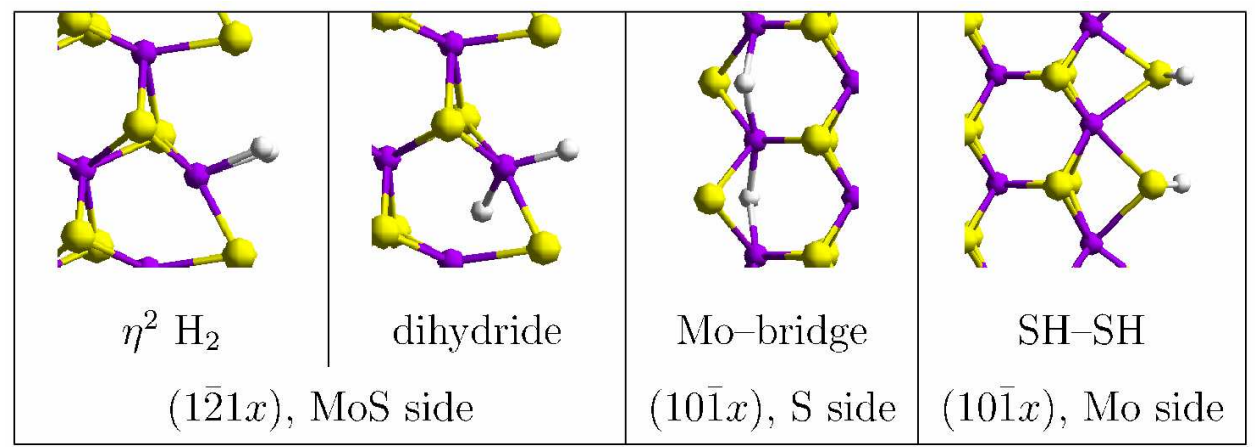

Figure 26: Geometries tested with two $\mathrm{H}$ atoms adsorbed on the $\mathrm{MoS}_{2}$ edge.

Table 7: Adsorption energies and vibrational frequencies for some $2 \mathrm{H}-\left(\mathrm{MoS}_{2}\right)_{n}$ configurations. $\Delta E=\mathrm{E}\left(2 \mathrm{H}-\left(\mathrm{MoS}_{2}\right)_{n}\right)-\left[\mathrm{E}\left(\left(\mathrm{MoS}_{2}\right)_{n}\right)+\mathrm{E}\left(\mathrm{H}_{2}\right)\right]$

\begin{tabular}{ccccc}
\hline Edge & Side & Position & $\Delta \mathrm{E}$ & $v$ \\
\hline$(10 \overline{1} x)$ & S side & Mo-bridge & $-0.34 \mathrm{eV}$ & \\
& Mo side & SH-SH & $-0.06 \mathrm{eV}$ & \\
\hline$(1 \overline{2} 1 x)$ & MoS side & $\eta^{2}-\mathrm{H}_{2}$ & $-0.52 \mathrm{eV}$ & \\
& & $0.28 \mathrm{eV}$ & $2999.2 \mathrm{~cm}^{-1}$ \\
& dihydride & & $(\mathrm{H}-\mathrm{H})$ \\
& & & $1483.9 \mathrm{~cm}^{-1}$ \\
& & & $\left(\mathrm{Mo-H}_{2} \mathrm{asym}^{-1}\right.$ \\
& & & $838.4 \mathrm{~cm}^{-1}$ \\
\hline
\end{tabular}

\section{Thermodynamics of Hydrogen Adsorption}

The adsorption of hydrogen atoms onto the $\mathrm{MoS}_{2}$ edges at constant pressure and temperature is governed by the change in Gibbs free energy for the reaction.

$$
\Delta G=\Delta U+p \Delta V-T \Delta S
$$

Electronic structure calculations provide $\Delta U$, one important term in the sum. Because the adsorption energies are small, the other terms do play a part. The $p \Delta V$ term is also small. Because the volume of adsorbed $\mathrm{H}$ is small compared to $\mathrm{H}_{2}$ gas, the $p \Delta V$ term is equal to $-\frac{P V}{n}=-R T=-\frac{1}{40} \mathrm{eV}$ at room temperature, and $-0.05 \mathrm{eV}$ at $623 \mathrm{~K}$.

The entropy penalty expressed in $-T \Delta S$ is more substantial. Its value is around $0.79 \mathrm{eV}$ at $623 \mathrm{~K}$ and $1 \mathrm{~atm}$ [28] and $0.40 \mathrm{eV}$ at $298 \mathrm{~K}$ and $1 \mathrm{~atm}$ [40]. Any adsorption mechanism must overcome the entropy penalty. 


\section{Conclusions}

Multiple edges of $\mathrm{MoS}_{2}$ basal planes have been studied by the DFT method using a one-dimensional ribbon model. The $(10 \overline{1} x)$ edge was found to be the most stable, though the $(1 \overline{2} 1 x)$ edge was found to be only $0.09 \mathrm{eV}$ per edge $\mathrm{MoS}_{2}$ unit less stable. The relaxation of the edge atoms was found to be important in a description of the geometry and stability of the edges. For reference, a 2-D single sheet of $\mathrm{MoS}_{2}$ has also been modeled. The DOS of the 2-D sheet closely matched that measured by XPS [31], and the band structure was substantially identical to that previously calculated by the LAPW DFT method [2] and measured experimentally by Böker et al. [34].

The $(1 \overline{2} 1 x)$ edge was found slightly higher in energy than the most stable edge, and therefore is not expected to occur naturally. However, this edge does provide unsaturated Mo atoms which could be sites for surface reactivity. Mo atoms on one edge were only 3-coordinated, while those on the other edge were 5-coordinated. The "vertically cut" $(1 \overline{2} 10)$ edge was actually more stable than the $(10 \overline{1} 0)$ edge by about $0.5 \mathrm{eV}$ per unit cell, but both of these were less stable than the "inclined cut" edges.

The analysis of the electronic structure revealed surface states associated with the edges. In particular, the HOMO of the relaxed $(10 \overline{1} x)$ edge was a bonding surface state with effective mass of an electron $\sim 1.9$ times the mass of a free electron. This feature stands in contrast with the "normal" electronic structure of an infinite edge-free 2-D $\mathrm{MoS}_{2}$ sheet, in which the HOMO has the characteristic effective mass of a hole 4.1 times the mass of a free electron. Thus the edge state may be viewed as an electronic "wire," as has been suggested by Bollinger et al. for large $\mathrm{MoS}_{2}$ clusters deposited on gold [35].

In addition, small stoichiometric $\mathrm{MoS}_{2}$ cluster models were examined. These clusters were found to exhibit much the same relaxation behavior as the infinite edges, with the exception that the Mo atoms did not change coordination on the $(10 \overline{1} x)$ edge in the $\mathrm{Mo}_{7} \mathrm{~S}_{14}$ model as they did in the periodic $(10 \overline{1} x)$ edge. The calculated properties of the $\mathrm{MoS}_{2}$ monomer were in excellent agreement with experimental data and theoretical results of Liang and Andrews [3] regarding the $\mathrm{MoS}_{2}$ molecule isolated in solid argon, despite the different functionals, basis sets, and core potentials used, reinforcing confidence in both approaches. In the ground state ${ }^{3} \mathrm{~B}_{1}$, the experimental S-Mo-S angle $114 \pm 3^{\circ}$ [3] agrees well with the DFT calculations, $113.5^{\circ}$ [3] and $114.44^{\circ}$ (present work). The calculated Mo-S bond lengths also agree, $2.132 \AA$ [3] and $2.148 \AA$ (present work), as does the energy difference between the excited singlet and the ground state triplet, $\Delta E\left({ }^{1} \mathrm{~A}_{1}-{ }^{3} \mathrm{~B}_{1}\right)=0.61 \mathrm{eV}[3]$ and $0.56 \mathrm{eV}$ (present work).

The transition from high-spin molecule to zero-spin solid $\mathrm{MoS}_{2}$ is reflected in the difference between the singlet and the triplet state which was calculated to be just about zero in the $\left(\mathrm{MoS}_{2}\right)_{7}$ cluster. The singlet-triplet bandgaps per $\mathrm{MoS}_{2}$ unit were $0.53 \mathrm{eV}$ for the monomer, $0.03 \mathrm{eV}$ for the trimer, $0.01 \mathrm{eV}$ for the heptamer. In the bulk crystal, the 
singlet was more stable and the bandgap was calculated to be $+1.29 \mathrm{eV}$. The triplet spin density, which represents a combined density of the hole and the electron forming the triplet (or 'exciton'), results from spin coupling due to exchange interaction which stabilizes the electronic high-spin state in highly correlated systems. The net effect is an increased delocalization of spin density from Mo to $\mathrm{S}$ with increasing cluster size, as seen in Figure 16, and correspondingly weakened exchange-stabilization of the triplets. Based on these results, it is predicted that small $\left(\mathrm{MoS}_{2}\right)_{n}$ clusters $(n \leq 7)$ will be paramagnetic with chemical properties appropriate to their spin state.

All of the present results indicate that the high-energy edge surfaces and peripheral groups of atoms in small clusters undergo very large structural relaxations associated with significant energy stabilization. These phenomena no doubt play an important role in accessibility of reactive sites and in chemisorption of molecules that are activated for catalytic reactions on these sites.

Hydrogen on the $\mathrm{MoS}_{2}$ molecule formed a dihydride with a singlet spin, though the bare $\mathrm{MoS}_{2}$ is a triplet the $\mathrm{H}_{2}$ molecule is a singlet. In the most stable $\mathrm{H}_{2}-\mathrm{MoS}_{2}$ configuration, the $\mathrm{H}-\mathrm{H}$ separation was found to be $3.08 \AA$ and there was little electron density seen between the $\mathrm{H}$ atoms. There was a metastable $\eta^{2}-\mathrm{H}_{2}$ configuration with a triplet spin and an $\mathrm{H}-\mathrm{H}$ distance of only $0.8 \AA$, but the $\eta^{2}-\mathrm{H}_{2}$ energy was $0.65 \mathrm{eV}$ higher than the dihydride. As the ground state was formed, the $\sigma^{*}$ orbital of the approaching $\mathrm{H}_{2}$ molecule was mixed with the d orbitals, leading to the double occupancy of this antibonding orbital and subsequent activation of the $\mathrm{H}_{2}$.

As the size of the $\mathrm{MoS}_{2}$ cluster was increased, the spin tended more toward the singlet state seen in bulk $\mathrm{MoS}_{2}$. The edges of the $\mathrm{Mo}_{7} \mathrm{~S}_{14}$ cluster resembled some of the bulk edges, and both the dihydride and the $\eta^{2}-\mathrm{H}_{2}$ configurations were found. There was no indication of binding of the adsorbed $\mathrm{H}$ to the $\mathrm{S}$ atoms. Doping of the $\mathrm{MoS}_{2}$ molecule or even an $\mathrm{NbS}_{2}$ molecule with an alkali atom such as potassium reduced the activation energy for the formation of the dihydride. This alkali doping could be used on the solid $\mathrm{MoS}_{2}$ to increase the take-up of $\mathrm{H}_{2}$ on the edges.

The periodic edge calculations did show $\mathrm{H}-\mathrm{S}$ bonds. Single hydrogen atoms were adsorbed on sites neighboring both $\mathrm{S}$ and Mo atoms, with the strongest binding on the $(1 \overline{2} 1 x)$ edge on the second-row S atom (Figure $25,(1 \overline{2} 1 x)$ edge, S side, S2-top). With molecular hydrogen adsorption, the only dihydride configuration examined indicated an endothermic adsorption. Strong binding in an $\eta^{2}-\mathrm{H}_{2}$ configuration was found on the $(1 \overline{2} 1 x)$ edge, in contrast with previous reports of heteropolar or homopolar adsorption on $\mathrm{S}$ from previous studies.

The vibrational frequencies of hydrogen bound to $\mathrm{S}$ and Mo atoms were found to be quite distinct and provide a definitive way to determine the adsorption sites of the hydrogen. When hydrogen was bound to the lighter $\mathrm{S}$ atoms, the vibration frequency was about $2500 \mathrm{~cm}^{-1}$, while this same frequency was lowered to about $1800 \mathrm{~cm}^{-1}$ when the $\mathrm{H}$ was bound to Mo atoms. The exception was for adsorption on the basal plane $\mathrm{S}$ atoms, where the $\mathrm{H}$ atom was found to have a vibration frequency of $2036 \mathrm{~cm}^{-1}$. This is due to 
the sulfur being braced by its bonds with the molybdenum plane, giving it a slightly higher effective mass.

\section{References}

1. J. A. Spirko, M. L. Neiman, A. M. Oelker, K. Klier, Surf. Sci. 542 (2003) 192-204.

2. K. T. Park, J. S. Hess, K. Klier, J. Chem. Phys. 111 (1999) 1636.

3. B. Liang, L. Andrews, J. Phys. Chem. A 106 (2002) 6945.

4. W. Kohn, L. J. Sham, Phys. Rev. 140 (1965) A1133.

5. R. G. Parr, W. Yang, Density Functional Theory of Atoms and Molecules, Oxford University Press, New York, 1989.

6. J. P. Perdew, Y. Wang, Phys. Rev. B 45 (1992) 13244.

7. B. Delley, J. Chem. Phys. 92 (1990) 508.

8. J. P. Perdew, S. Burke, M. Ernzerhof, Phys. Rev. Lett. 77, 3865.

9. P. Blaha, K. Schwarz, G. K. H. Madsen, D. Kvasnicka, J. Luitz, WIEN2k, an Augmented Plane Wave + Local Orbitals Program for Calculating Crystal Properties (Karlheinz Schwarz, Techn. Univesität Wien, Austria), 2001. ISBN 39501031-1-2.

10. D. Becke, Phys. Rev. A 38 (1988) 3089.

11. J. P. Perdew, Phys. Rev. B 33 (1986) 8822.

12. Spartan, version 5.1.3, Wavefunction, Inc., Irvine, CA, 1998.

13. R. Fletcher, Practical Methods of Optimization, Wiley, New York, 1990.

14. J. J. P. Stewart, J. Comp. Chem. 10 (1989), 209.

15. Q. Ma, K. Klier, H. Cheng, J. W. Mitchell, K. S. Hayes, J. Phys. Chem. B 104 (2000), 10618.

16. Q. Ma, K. Klier, H. Cheng, J. W. Mitchell, K. S. Hayes, J. Phys. Chem. B 105 (2001), 2212.

17. Q. Ma, K. Klier, H. Cheng, J. W. Mitchell, K. S. Hayes, J. Phys. Chem. B 105 (2001), 9230.

18. Q. Ma, K. Klier, H. Cheng, J. W. Mitchell, J. Phys. Chem. B 106 (2002), 10121.

19. H. Cheng, J. W. Mitchell, K. S. Hayes, M. Neurock, C. Smead, Q. Ma, K. Klier, NATO Advanced Studies Serios, Kluwer Academic 2002, 385.

20. L. S. Byskov, J. K. Nørskov, B. S. Clausen, H. Topsøe, Catal. Lett. 64 (2000) 95-99.

21. P. Raybaud, J. Hafner, G. Kresse, S. Kasztelan, H. Toulhoat, J. Catal. 190 (2000) 128.

22. R. R. Chianelli, G. Berhault, P. Raybaud, S. Kasztelan, J. Hafner, H. Toulhoat, Appl. Catal. A: General 227 (2002) 83.

23. P. Raybaud, J. Hafner, G. Kresse, S. Kasztelan, H. Toulhoat, J. Catal. 189 (2000) 129.

24. P. Raybaud, J. Hafner, G. Kresse, H. Toulhoat, Surf. Sci. 407 (1998) 237.

25. S. Cristol, J. F. Paul, E. Payen, D. Bougeard, S. Clémendot, F. Hutschka, J. Phys. Chem. B 104 (2000) 11220.

26. H. Schweiger, P. Raybaud, G. Kresse, H. Toulhoat, J. Catal. 207 (2002) 76.

27. S. Lobos, A. Sierraalta, F. Ruette, E. N. Rodríguez-Arias, J. Mol. Catal. A: Chemical 192 (2003) 203. 
28. S. Cristol, J. F. Paul, E. Payen, D. Bougeard, S. Clémendot, F. Hutschka, J. Phys. Chem. B 104 (2002) 5659.

29. H. Orita, K. Uchida, N. Itoh, J. Mol. Catal. A: Chemical 195 (2003) 173.

30. L. S. Byskov, J. K. Nørskov, B. S. Clausen, H. Topsøe, J. Catal. 187 (1999) 109.

31. K. T. Park, M. Richards-Babb, J. S. Hess, J. Weiss, K. Klier, Phys. Rev. B 54 (1996) 5471.

32. J. H. Scofield, J. Electron Spectrosc. Relat. Phenom. 8 (1981) 129.

33. M. Richards-Babb, Ph.D. thesis, Lehigh University (1992).

34. T. Böker， R. Severin， A. Müller，C. Janowitz， R. Manzke，D. Voß，P. Krüger, A. Mazur, J. Pollmann, Phys. Rev. B 64 (2001) 235305.

35. M. V. Bollinger, J. V. Lauritsen, K. W. Jacobsen, J. K. Nørskov, S. Helveg, F. Besenbacher, Phys. Rev. Lett. 87 (2001) 196803.

36. S. G. Davison, J. Grindlay, Surf. Sci. 11 (1968) 99-110.

37. J. A. Wilson, A. D. Yoffe, Adv. Phys. 18 (1969) 193.

38. G. J. Kubas, J. Organometallic Chem. 635 (2001) 37-68.

39. D. R. Lide (Ed.), CRC Handbook of Chemistry and Physics, 72nd Edition, CRC Press, Boca Raton, FL, 1992.

40. J. D. Fast, Entropy, 2nd Edition, Taylor \& Francis, 1969. 